\title{
Met is required for oligodendrocyte progenitor cell migration in Danio rerio
}

\author{
Maria F. Ali, ${ }^{1}$ Andrew J. Latimer, ${ }^{1}$ Yinxue Wang, ${ }^{2}$ Leah Hogenmiller, ${ }^{1}$ Laura Fontenas, ${ }^{1}$ Adam J. Isabella, ${ }^{3}$ Cecilia B. Moens (D) , \\ Guoqiang Yu (D) , ${ }^{2}$ and Sarah Kucenas (D) ${ }^{1, *}$ \\ ${ }^{1}$ Department of Biology, University of Virginia, Charlottesville, VA 22904, USA \\ 2Department of Electrical and Computer Engineering, Virginia Polytechnic Institute and State University, Arlington, VA 22203, USA \\ ${ }^{3}$ Division of Basic Sciences, Fred Hutchinson Cancer Research Center, Seattle, WA 98109, USA \\ *Corresponding author: Physical \& Life Sciences Building, Rm 312, PO Box 400328, Charlottesville, VA 22904-4328, USA. Email: sk4ub@virginia.edu
}

\begin{abstract}
During vertebrate central nervous system development, most oligodendrocyte progenitor cells (OPCs) are specified in the ventral spinal cord and must migrate throughout the neural tube until they become evenly distributed, occupying non-overlapping domains. While this process of developmental OPC migration is well characterized, the nature of the molecular mediators that govern it remain largely unknown. Here, using zebrafish as a model, we demonstrate that Met signaling is required for initial developmental migration of OPCs, and, using cell-specific knock-down of Met signaling, show that Met acts cell-autonomously in OPCs. Taken together, these findings demonstrate in vivo, the role of Met signaling in OPC migration and provide new insight into how OPC migration is regulated during development.
\end{abstract}

Keywords: oligodendrocyte progenitor cells; Met receptor; glial migration; spinal cord development; zebrafish

\section{Introduction}

During vertebrate spinal cord development, oligodendrocyte progenitor cells (OPCs) are specified from ventral gliogenic precursors (Lu et al. 2000; Dimou et al. 2008; Ravanelli et al. 2018). Immediately following specification, these cells undergo a process termed tiling where they actively disperse throughout the spinal cord, ultimately forming nonoverlapping domains with neighboring OPCs (Kirby et al. 2006; De Biase et al. 2017). Although tiling is a behavior that has been extensively studied in the context of neuronal development, very few studies have characterized these events in glia, although this behavior is commonly reported in the literature (Cameron and Rao 2010; Grueber and Sagasti 2010; Villar-Cerviño et al. 2013; López-Hidalgo et al. 2016; De Biase et al. 2017; Nichols et al. 2018). The process of OPC tiling is comprised of four main cellular behaviors: migration, proliferation, cell death, and contact-mediated repulsion (Kirby et al. 2006; Hughes et al. 2013; Huang et al. 2020). Though the phenomenon of OPC tiling is well-described, the molecular mediators of this process remain largely unknown. What has been identified are a number of chemoattractant or chemorepellent molecules that influence OPC migration (Spassky et al. 2002; Tsai et al. 2002; Jarjour et al. 2003; Chu et al. 2017). In this study, we sought to identify, in an unbiased manner, molecular mediators that govern the initial migration of OPCs during developmental tiling. Using an unbiased, small molecule screen, we identified several pathways, including Met signaling, as essential mediators of OPC migration.
Previously, in vitro studies revealed that hepatocyte growth factor (Hgf), the ligand for Met, acts as a mitogenic signal for OPCs (Lalive et al. 2005; Ohya et al. 2007). Met, also known as scatter factor receptor or Hgf receptor, is a widely studied receptor tyrosine kinase that is involved in a number of morphogenetic processes during embryogenesis, including regulating cellular migration and motility (Soriano et al. 1995; Birchmeier and Gherardi 1998; Prat et al. 1998; Viticchiè et al. 2015; Zhang and Babic 2015). In particular, OPCs in culture, upon application of Hgf, exhibit increased migration and proliferation (Lalive et al. 2005; Ohya et al. 2007). Additionally, it is well documented that OPCs express the c-Met receptor (Kilpatrick et al. 2000; Lalive et al. 2005; Ohya et al. 2007; Mela and Goldman 2013). Though these studies established foundational work supporting Met as a possible mediator of OPC migration, further investigation into the role of Met signaling in regulating developmental OPC migration in vivo were impeded because mouse Met mutants are embryonic lethal.

In order to study the role of Met signaling in vivo, we utilized zebrafish as a vertebrate model. Because zebrafish embryos receive maternal mRNAs, including met mRNA from their mother (Latimer and Jessen 2008), zebrafish met mutants successfully complete embryogenesis and can therefore be used to investigate later developmental processes, including OPC migration. In fact, many recent studies have used zebrafish embryos and larvae lacking Met function to study a number of development processes including motor axon targeting and migratory muscle 
precursor migration (Nord et al. 2019; Talbot et al. 2019; Isabella et al. 2020).

Here, we describe the identification of the Met receptor as an essential mediator of OPC migration. Using a combination of pharmacological and genetic manipulation with in vivo, timelapse imaging and a new software to analyze migration dynamics, we demonstrate that Met signaling is required for the initial, dorsal migration of OPCs during development of the vertebrate spinal cord. Furthermore, by utilizing cell-specific dominant negative Met (DNmet) constructs, we show that Met acts cellautonomously within OPCs. Together, our results demonstrate that Met signaling regulates initial OPC migration during developmental tiling.

\section{Materials and methods Zebrafish husbandry}

All animal studies were approved by the University of Virginia Institutional Animal Care and Use Committee. Zebrafish strains used in this study were: $\mathrm{AB}^{*}$, met $\mathrm{t}^{\text {uva38}}, \mathrm{met}^{\mathrm{fh534}}, \mathrm{Tg}$ (sox10 (4.9):tagrfp) uva5, $\mathrm{Tg}$ (olig2:egfp) ${ }^{\text {uu12 }}$ (Shin et al. 2003); $\mathrm{Tg}$ (sox10(7.2):

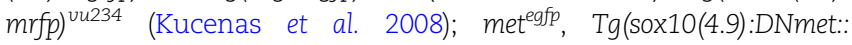

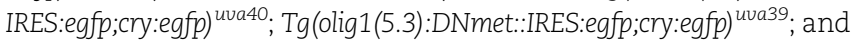
hgfa $^{\text {fh528 }}$ (Isabella et al. 2020). Table 1 denotes abbreviations used for each mutant and transgenic line. Embryos were raised at $28.5^{\circ} \mathrm{C}$ in egg water and staged by hours or days post-fertilization (hpf and dpf, respectively) (Kimmel et al. 1995). Embryos of either sex were used for all experiments. Phenyl-thiourea (PTU) (0.004\%) (Sigma) in egg water was used to reduce pigmentation for imaging. Stable, germline transgenic lines were used in all experiments.

\section{Generation of transgenic lines}

All constructs were generated using the Tol2kit Gateway-based cloning system (Kwan et al. 2007). Vectors used for making the expression constructs were p5E-sox10(-4.9) (Carney et al. 2006), p5Eolig1(-5.3) (this paper), pME-DNmet (this paper), p3E-IRESEGFPpA, and pDestTol2pA2 destination vector (Kwan et al. 2007).

To generate p5E-olig1, we amplified $5.3 \mathrm{~kb}$ of sequence immediately upstream of the olig1 gene (Ensembl: ENSDARG 00000040948) from wildtype genomic DNA using the following primers: forward 5'-GTATGAAGCCTCTTGGCACAG-3' and reverse 5'-CTGAAAAAAGATATTCAGAGAACATGG-3', as previously described (Auer et al. 2018). The resulting PCR product was subcloned into pENTR ${ }^{\mathrm{TM}} 5^{\prime}$-TOPO (Invitrogen) to generate a p5E entry for Gateway cloning and was verified by sequencing.

To generate pME-DNmet, we used the Q5 Site-Directed Mutagenesis Kit (NEB) and generated site-specific mutations in met CDNA, which we generated using RT-PCR as described below, using the following primers: forward 5'-CAACATCGACAA
AATGACACCCTTCCCCTCTCTCATATCATCTCAG-3' and reverse, $5^{\prime}$-ACGAAGGTGGTGTTCAGGAGGATGAAGTGCTCTCCGCTGAA GC-3'. We confirmed the mutations using sequencing, then subcloned the cDNA containing the DNmet mutations into pME-MCS to generate a pME entry for Gateway cloning. p5E, pME, and p3E-IRES-EGFPpA vectors were ligated into destination vectors through LR reactions (Ashton et al. 2012). Final constructs were amplified, verified by restriction digest, and sequenced to confirm the insertions. To generate stable transgenic lines, plasmid DNAs were microinjected at a concentration of $25 \mathrm{ng} / \mu \mathrm{L}$ in combination with $10 \mathrm{ng} / \mu \mathrm{L}$ Tol2 transposase mRNA at the one-cell stage and screened for founders (Kawakami 2004).

The met $\mathrm{fh}^{\text {f55Tg }}$ enhancer trap line was generated using the CRISPR/Cas9-mediated knock-in strategy described in (Kimura et al. 2014). One nanoliter of a cocktail of the following components was injected into one-cell stage embryos: $66.6 \mathrm{ng} / \mu \mathrm{L}$ each of 3 CRISPR guide RNAs targeting within or just upstream of the met 5' UTR (target sequences GGTCTCGGGATGGGATGCGA, GGTTCTCTCCGCAAACGCTG, and GGGTAAGCGGGTTCGCTGAT), $200 \mathrm{ng} / \mu \mathrm{L}$ Mbait gRNA, $20 \mathrm{ng} / \mu \mathrm{L}$ Mbait-hsp70-GFP plasmid, $800 \mathrm{ng} / \mu \mathrm{L}$ Cas9 protein (PNABio \#CP02). Founders were screened for GFP expression replicating the known met expression pattern.

\section{Generation of mutant lines}

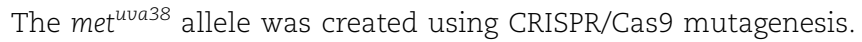
Using CHOPCHOP, we generated a gRNA specific to exon 2 of the zebrafish met coding sequence with a target sequence of: AGTGCATTTACTCTGAGCGA (Labun et al. 2019). Using this synthesized gRNA, we injected 1-2 nl of the sgRNA (200-400 ng/ $\mu \mathrm{L})$ with Cas9 protein $(500 \mathrm{ng} / \mu \mathrm{L})$ into one-cell embryos and grew up potential founders to adulthood. We then outcrossed putative founders and screened for frameshift mutations in their offspring and identified a founder with a mutation that resulted in a 16 base pair insertion with a one base pair insertion at nucleotide 890 of the coding sequence and a 15 base pair insertion at nucleotide 895 of the coding sequence, resulting in a frameshift at amino acid 297 and a premature stop at amino acid 328. The met $\mathrm{fh534}^{\mathrm{fl}}$ allele was made using a standard CRISPR/Cas9 mutagenesis protocol (Talbot and Amacher 2014). One cell stage embryos were injected with $100 \mathrm{pg}$ each of 2 CRISPR guide RNAs (target sequences GGTTCTGGCCATCTGGCTCG and GGCTTCGGCTG CGTGTTTCA) and 500 pg Cas9 protein, and F1 mutant animals were identified by sequencing. et $^{f h 534}$ is a 25 base pair deletion starting at nucleotide 3275 of the coding sequence, resulting in a frameshift at amino acid 1093 and a premature stop at amino acid 1105. For both the met ${ }^{\text {uva38 }}$ and met $\mathrm{f}^{\text {fh534 }}$ alleles, all studies were done on embryos and larvae derived from outcrossed adults from the F3 generation and beyond.

Table 1 Zebrafish lines used in this study and their genotypes

\begin{tabular}{|c|c|c|}
\hline Full name & Abbreviation & Reference \\
\hline $\begin{array}{l}\text { AB* } \\
\text { Tg(olig2:egfp) vu12 } \\
\text { Tg(sox10(4.9):tagrfp) uva5 } \\
\text { met fh555Tg } \\
\text { Tg(sox10(7.2):mrfp) vu234 } \\
\text { met } \\
\text { Tg (soxa38 } \\
\text { Tg(olig1(5.3):DNmet::IRES:egfp;cry:egfp) }{ }^{\text {uva39 }} \\
\text { hgfafh528 } \\
\text { met }\end{array}$ & $\begin{array}{l}\text { Wildtype } \\
\text { olig2:egfp } \\
\text { sox10:tagrfp } \\
\text { met } \text { egfp }^{\text {sox10:mrfp }} \\
\text { sotuvis/uva38 } \\
\text { met } \\
\text { sox10:DNmet } \\
\text { olig1:DNmet } \\
\text { hgfa }^{\text {fh528/fh528 }} \\
\text { met } \\
\text { fh534/fh534 }\end{array}$ & $\begin{array}{c}\text { Shin et al. (2003) } \\
\text { Zhu et al. (2019) } \\
\text { This paper } \\
\text { Kucenas et al. (2008) } \\
\text { This paper } \\
\text { This paper } \\
\text { This paper } \\
\text { Isabella et al. (2020) } \\
\text { This paper }\end{array}$ \\
\hline
\end{tabular}




\section{In vivo imaging}

Embryos were anesthetized with 0.01\% 3-aminobenzoic acid ester (Tricaine), immersed in $0.8 \%$ low-melting point agarose and mounted in glass-bottomed $35 \mathrm{~mm}$ Petri dishes (Electron Microscopy Sciences). After mounting, the Petri dish was filled with egg water containing PTU and Tricaine. A 40x water objective (NA = 1.1) mounted on a motorized Zeis AxioObserver Z1 microscope equipped with a Quorum WaveFX-X1 (Quorum Technologies) or Andor CSU-W1 (Oxford Instruments) spinning disc confocal system was used to capture all images. Images were imported into MetaMorph (Molecular Devices) and/or ImageJ. Time-lapse images were analyzed using cell tracking software as previously described in (Wang et al. 2018). All images were then imported in Adobe Photoshop and Illustrator. Adjustments were limited to levels, contrast, and cropping.

\section{Cell tracking software}

For quantification and motility analysis of OPCs, we developed an automated software to detect and track motile cells in timelapse imaging experiments of olig2:egfp embryos and larvae (Wang et al. 2018). To correct for photobleaching, we normalized fluorescence intensity to an identical mean and an identical variance at all-time points and to account for long-term image shift due to larval growth, we used global image registration. For intraframe detection and segmentation of all cells, we applied the cell detection algorithm SynQuant to map the second-order derivative transformed intensity (Wang et al. 2020). The undersegmentation and over-segmentation in intra-frame detection were then corrected by imposing temporal consistency of segmentation, which was modeled as an optimization problem. Rapid motion was detected by testing regional intensity change and to link the detections, we adapted our established algorithm muSsP to a mixed motion model form (Wang et al. 2019).

The motion patterns of all obtained traces were then analyzed to obtain a quantification of cell motility and to distinguish OPCs from other cells in the field of view. The distance traveled was calculated by adding up the magnitude of displacement between any two consecutive time points and the instantaneous velocity of any olig2 ${ }^{+}$OPC was approximated by the average velocity between two consecutive time points, while the instantaneous speed was the magnitude of it. The overall average speed was the distance traveled divided by time period and we identified OPCs from neighboring cells by hypothesis testing on the instantaneous velocity. Assuming the majority of cells in the field of view were not moving, the null distribution was learned by fitting a multivariate Gaussian distribution to all instantaneous velocity values of the obtained traces. Any cell whose trace once had significantly high instantaneous velocity was identified as an OPC.

\section{Whole-mount immunohistochemistry}

Dechorionated embryos and larvae were fixed with 4\% PFA for $1 \mathrm{~h}$ at room temperature (RT), washed in PBSTX (1\% Triton X-100, $1 \times$ PBS) for $5 \mathrm{~min}$, followed by a $5 \mathrm{~min}$ wash with DWTx $(1 \%$ Triton $\mathrm{X}-100$ in $\mathrm{DH}_{2} \mathrm{O}$ ), then permeabilized in acetone at RT for $5 \mathrm{~min}$ and at $-20^{\circ} \mathrm{C}$ for $10 \mathrm{~min}$, followed by a $5 \mathrm{~min}$ wash with PBSTx. Embryos were then blocked in 5\% goat serum/PBSTx for $1 \mathrm{~h}$, incubated in primary antibody with $5 \%$ goat serum/PBSTx for $1 \mathrm{~h}$ at $\mathrm{RT}$ and overnight at $4^{\circ} \mathrm{C}$. Embryos were washed extensively with PBSTx at RT and incubated in secondary antibody overnight at $4^{\circ} \mathrm{C}$. After antibody incubation, embryos were washed extensively with PBSTx and stored in PBS at $4^{\circ} \mathrm{C}$ until imaging. The following antibodies were used: rabbit anti-Sox10 (1:5000) (Binari et al. 2013), Alexa Fluor 647 goat anti-rabbit IgG(H+L) (1:1000; ThermoFisher). Embryos were mounted in glass-bottomed Petri dishes for imaging as described above.

\section{Immunohistochemistry on sections}

Embryos and larvae were fixed in 4\% PFA for $2 \mathrm{~h}$ at RT. After fixation, the embryos were sectioned by embedding them in $1.5 \%$ agarose/30\% sucrose and frozen in 2-methylbutane chilled by immersion in liquid nitrogen. We collected $20 \mu \mathrm{m}$ transverse sections on microscope slides using a cryostat microtome. Sections were rehydrated in PBS for $1 \mathrm{~h}$ and blocked with $5 \%$ goat serum/ PBS for $1 \mathrm{~h}$ at RT. Primary antibody incubation was done overnight at $4^{\circ} \mathrm{C}$. Secondary antibody incubation was done for $2 \mathrm{~h}$ at RT. Antibodies used were: rabbit anti-Sox10 (1:5000) (Binari et al. 2013), mouse 3D4 anti-Met (1:100; ThermoFisher), Alexa Fluor 647 goat anti-rabbit IgG(H+L) (1:1000), and Alexa Fluor 568 goat anti-mouse IgG(H+L) (1:1000). Sections were covered with AquaPoly/Mount (Polysciences). A $63 \times$ oil objective ( $\mathrm{NA}=1.4$ ) mounted on a motorized Zeiss AxioObserver Z1 microscope equipped with a Quorum WaveFX-X1(Quorum Technologies) or Andor CSU-W1 (Andor Oxford Instruments) spinning disc confocal system was used to capture all images. Images were imported into Image $J$ and Adobe Photoshop and Illustrator. Adjustments were limited to levels, contrast, and cropping.

\section{Data collection}

Because met mutant embryos and larvae had no morphological phenotypes, all of our studies were conducted blind. Data was collected and analyses completed prior to individual embryo/ larva genotyping. Studies using sox10:DNmet and olig1:DNmet embryos and larvae were pre-screened for expression of the cry:egfp reporter and therefore, the genotype of the animals used was known to the investigator prior to data analysis. For quantification, all data were collected between somite 10 and 20. Quantifications of cells in serial cryosections were taken from 10 sequential $20 \mu \mathrm{m}$ sections and reported as a total value in $200 \mu \mathrm{m}$. For identification and quantification of dorsal, ventral, or pMN domain OPCs, we divided transverse images of the spinal cord into four equal sections. Any OPC in the top two sections was considered a dorsal OPC. OPCs in the third section from the top were considered pMN domain OPCs. OPCs in the bottom quarter of the spinal cord were considered ventral OPCs.

\section{Chemical treatments}

In our initial small molecule screen, dechorionated olig2:egfp larvae were treated with $10 \mu \mathrm{m}$ kinase inhibitor in $1 \%$ DMSO in PTU egg water from 24 to $76 \mathrm{~h}$ post-fertilization (hpf). Kinase inhibitors used were 1 of 430 kinase inhibitors from the L1200 Kinase Inhibitor Library (Selleck Chem), MK-2461 (Selleck Chem), or Trichostatin-A (TSA) (Selleck Chem). Control siblings were treated with $1 \%$ DMSO in PTU egg water. The small molecule screen was conducted in triplicate.

For the EdU incorporation assay, larvae were treated with $0.4 \mathrm{mM}$ EdU in $4 \%$ DMSO from 70 to $74 \mathrm{hpf}$ in PTU egg water at $28.5^{\circ} \mathrm{C}$ then fixed for $1 \mathrm{~h}$ in $4 \%$ PFA at RT. Larvae were washed for 5 min with $1 \mathrm{X}$ PBSTX, 5 min in DWTX, then permeabilized with cold acetone for $10 \mathrm{~min}$ at $-20^{\circ} \mathrm{C}$ and stained for EdU using the Click-it EdU Cell Proliferation kit for Imaging with Alexa Fluor 647 dye (ThermoFisher), as detailed in the kit protocol. Click-it reaction was performed for $1 \mathrm{~h}$ at RT and thoroughly washed overnight with PBSTX prior to imaging. 


\section{Genotyping}

Genomic DNA was extracted using HotSHOT (hot sodium hydroxide and tris) and PCR was performed using GoTaq green master mix (Promega) (Truett et al. 2000). The primers used for genotyping met $^{\text {uva38 }}$ are as follows: forward 5'-ATCGTACGCATGTGT TCTTCAG-3' and reverse 5'-TGATGTCCGTGATGGAGATAAG-3'. The primers used for genotyping met $f^{f 534}$ are as follows: forward 5'-AATCTCTGCCATGTTTTCCTGT- $3^{\prime}$ and reverse 5'-AGTCCAA AACTATCCCAAGCAA-3'. The primers used for genotyping hgfa $a^{\text {fh528 }}$ are as follows: forward $5^{\prime}$-TGTAAAACGACGGCCAGTC TCCATGAAGACCTTAGTTGGC-3' and reverse 5'-GTGTCTTGT GAACAGACCACACACACTGA-3'.

\section{RT-PCR}

mRNA was extracted and cDNA synthesized as described previously (Peterson and Freeman 2009) with the use of a RNA easy Mini kit (Qiagen) and High-capacity cDNA Reverse Transcription kit (Applied Biosystems) according to manufacturer instructions. Equal amounts of mRNA were used for cDNA synthesis, and PCR was performed using GoTaq green master mix (Promega). Primers used were: ef $1 \alpha$ forward: 5'-GAGACTGGTGTCCTCAAGCC$3^{\prime}$ and reverse: 5'-CCAACGTTGTCACCAGGAGT-3', and met ${ }^{u v a 38}$ forward: 5'-ATCGTACGCATGTGTTCTTCAG-3' and reverse: 5'TGATGTCCGTGATGGAGATAAG-3'.

\section{Statistical analysis}

GraphPad PRISM 9 software was used to plot data and perform statistical analyses. Pairwise comparison P-values involving only two groups were calculated using a Student's two-tailed t-test. Pairwise comparison P-values involving more than two groups were calculated using a one-way ANOVAs followed by Dunnett's Multiple Comparison tests or Tukey's Multiple Comparison test. The data in plots and the text are presented as means \pm SEM.

\section{Results \\ Met inhibition impairs developmental OPC migration}

While the phenomena of OPC migration and tiling are well known, very few mediators of this process have been identified. Therefore, to identify molecular mediators of OPC tiling, we conducted an unbiased kinase inhibitor screen in zebrafish embryos and larvae. To do this, we treated olig2:egfp zebrafish embryos, where olig2 regulatory sequences drive expression of GFP in motor neurons and oligodendrocyte lineage cells (OLCs), with 1\% DMSO as a control, $0.2 \mu \mathrm{M}$ TSA in $1 \%$ DMSO as a control, or $10 \mu \mathrm{M}$ of 1 of 430 kinase inhibitors from the Selleck Kinase Inhibitor Library in 1\% DMSO. We used TSA as a positive control because it is a histone deacetylase inhibitor that blocks OPC specification and therefore, embryos treated with this compound would show significantly reduced OPC migration into the dorsal spinal cord (Cunliffe and Casaccia-Bonnefil 2006) (Figure 1, A and B). We treated the embryos from $24 \mathrm{~h}$ post-fertilization (hpf), which is prior to OPC specification, until $76 \mathrm{hpf}$, which is during the middle-to-late migratory phase of these cells. By treating prior to OPC specification, we sought to identify molecular mediators that affect OPC migration, but do not block OPC specification. If we observed a complete absence of OPC migration, similar to that which occurs in the presence of TSA, then it is possible that the small molecule affected either OPC specification or migration. However, if we saw defects in migration, but there were still OPCs present, then we knew the kinase inhibitor likely did not affect specification. At $76 \mathrm{hpf}$, we first screened drug-treated larvae to confirm that overall larval morphology was indistinguishable compared to DMSO-treated larvae. We then individually screened drug-treated larvae for changes in OPC migration by looking for either an increase or decrease in the number of olig2 ${ }^{+}$ cells in the dorsal spinal cord compared to DMSO-treated controls (Figure 1B). From our screen of 430 kinase inhibitors, we identified 35 compounds that resulted in decreased numbers of OPCs in the dorsal spinal cord and 19 compounds that resulted in increased numbers of OPCs in the dorsal spinal cord. One exciting "hit" was in larvae treated with MK2461, a c-Met inhibitor, in which we observed a decrease in the number of OPCs in the dorsal spinal cord.

To confirm the reduction in dorsal OPCs we observed during MK2461 treatment, we used in vivo, time-lapse imaging in 55 hpf olig2:egfp larvae treated with MK2461 from $24 \mathrm{hpf}$ on (Figure 1C). In these movies, we see a significant reduction in the number of dorsally migrating OPCs (Figure 1C). However, we did observe active OPC migration in the ventral spinal cord, indicating that OPCs are specified but exhibit migration defects (Figure 1C). For quantification and motility analyses of OPCs, we developed an automated software to detect and track motile olig2 ${ }^{+}$OPCs distinct from olig2 ${ }^{+}$cells in $\mathrm{pMN}$ domain of olig2:egfp larvae, which contains a mixture of migratory OPCs and non-migratory motor neurons and precursors (Supplementary Movie S1) (Wang et al. 2018). Using this cell tracking software, we assessed the behaviors of OPCs in time-lapse movies from 55 to 74 hpf. From these analyses, we found that the number of migratory OPCs was significantly reduced in MK2461-treated larvae compared to control larvae (Figure 1D, Supplementary Movies S2 and S3). Specifically, we observed significantly reduced numbers of dorsally, but not ventrally, migrating OPCs in MK2461-treated larvae compared to DMSO-treated controls in a 3-somite window (Figure 1, E and F).

We next wanted to determine if treatment with MK2461 altered other aspects of OPC migration, including velocity and distance traveled. To do this, we used our cell-tracking software to analyze our time-lapse movies, like those shown in Figure 1C. Overall, MK2461-treatment did not alter velocity or distance traveled when looking at all OPCs compared to control larvae (Supplementary Figure S1, A and B). However, the dorsally migrating, and not ventrally migrating, OPCs traveled a shorter distance in MK2461-treated larvae compared to DMSO-treated control larvae (Supplementary Figure S1, C and E). The average velocity of migration for dorsally and ventrally migrating OPCs was not affected by MK2461 treatment (Supplementary Figure S1, $\mathrm{D}$ and F). Taken together, we observed that treatment with MK2461 resulted in a reduction in the number of OPCs that migrate dorsally during development and, therefore, the Met receptor is a likely mediator of OPC dynamics during development.

\section{Zebrafish OPCs express the Met receptor}

Previous in vitro studies of mouse and rat OPCs used antibody labeling to show that OPCs express the Met receptor (Kilpatrick et al. 2000; Lalive et al. 2005; Ohya et al. 2007; Mela and Goldman 2013). Therefore, we wanted to determine if zebrafish OPCs also expressed Met. To investigate Met expression in OLCs, we used the c-Met 3D4 antibody to label $\mathrm{Met}^{+}$cells in 3 dpf sox10:tagrfp;olig2:egfp zebrafish larvae. We then imaged transverse sections through the spinal cord of antibody-labeled larvae and observed $\mathrm{Met}^{+} / \mathrm{sox} \mathrm{O}^{+} / \mathrm{olig} 2^{+}$cells in the spinal cord (Figure 2A). Interestingly, not all sox $10^{+} /$olig $^{+}$cells were $\mathrm{Met}^{+}$, indicating that there are populations of both $\mathrm{Met}^{+}$and $\mathrm{Met}^{-}$OLCs (Figure 2A). 
A

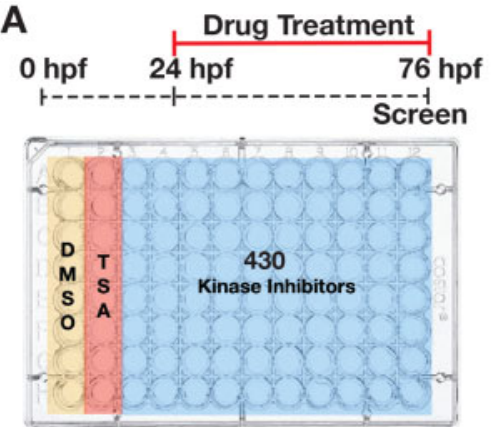

C
B

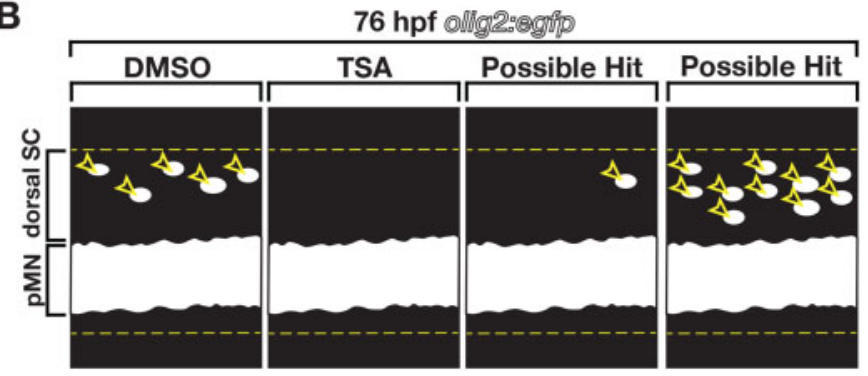

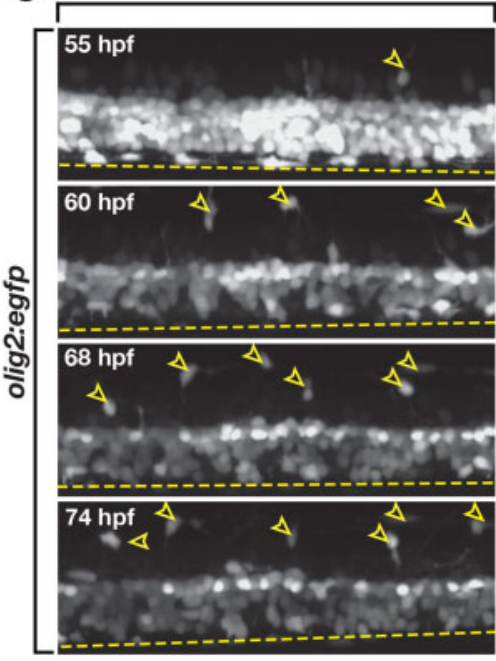

\section{D}

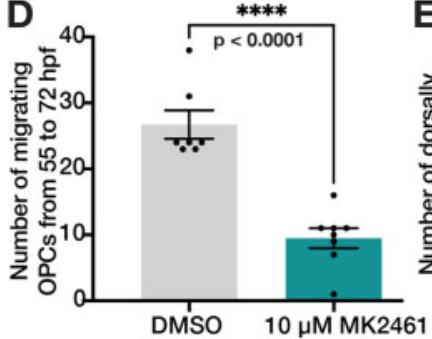

E

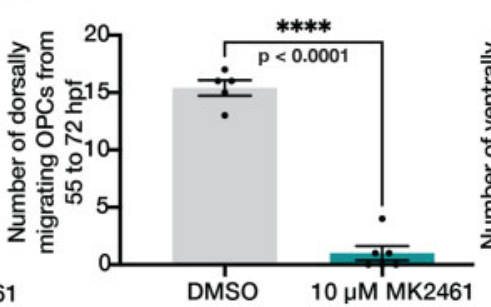

$10 \mu \mathrm{M}$ MK2461

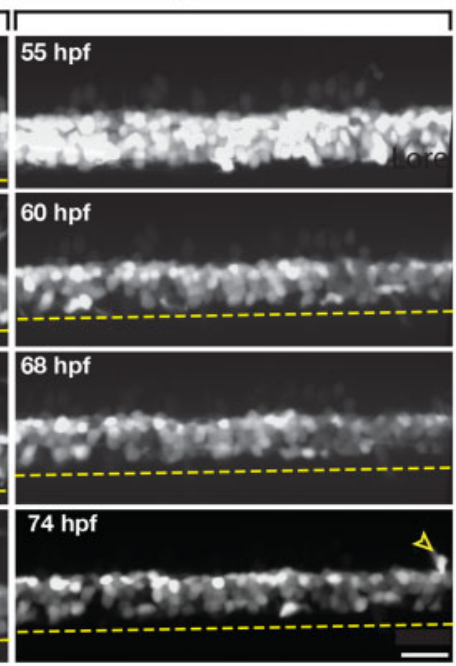

F

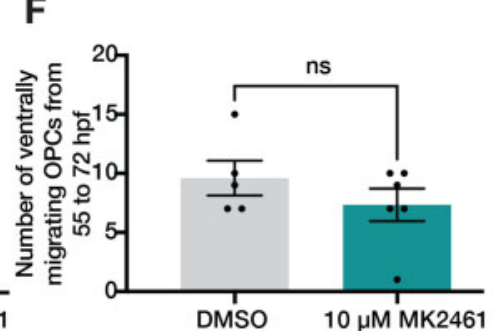

Figure 1 Kinase inhibitor screen identifies Met as mediator of dorsal OPC migration. (A) Schematic of kinase inhibitor screen and treatment paradigm that tested 430 kinase inhibitors for developmental OPC migration defects. Trichostatin A (TSA), which inhibits OPC specification, was used as a positive control. (B) Cartoon of a lateral view of $76 \mathrm{hpf}$ olig2:egfp larvae spinal cord showing DMSO (negative control), TSA (positive control), and examples of possible hits: reduced OLCs in the dorsal spinal cord (SC) and increased OLCs in the dorsal SC. pMN denotes pMN domain. Yellow dashed lines mark the boundaries of the spinal cord and open yellow arrowheads mark dorsal OLCs. (C) Images taken from $18 \mathrm{~h}$ time-lapse movies of DMSO and MK2461treated $55 \mathrm{hpf}$ olig2:egfp zebrafish larvae. Yellow open arrowheads denote dorsally migrating OPCs. Yellow dashed line denotes ventral edge of the spinal cord. (D-F) Quantifications taken from time-lapse movies of DMSO $(n=7)$ and MK2461-treated $(n=7)$ larvae in $(C)$. Mean with SEM. Student's t-test was used in D-F. Scale bar, $20 \mu \mathrm{m}$.

To confirm these findings, we used a new enhancer trap transgenic line, metegfp, where eGFP is under met regulation due to CRISPR-targeted insertion immediately upstream of the endogenous met gene, to assess met expression in OLCs from 48 to $96 \mathrm{hpf}$ (Kimura et al. 2014). To label OLCs, we labeled met ${ }^{\text {egfp }}$ embryos and larvae with our Sox10 antibody (Figure 2B). We then imaged the spinal cord and quantified the number of $\mathrm{met}^{+} / \mathrm{Sox} 10^{+}$cells. We found that at $48 \mathrm{hpf}$, just prior to OPC migration, a large percentage of Sox $10^{+}$OPCs were met $^{+}$(78.95\%) (Figure 2C). By 55 hpf, only $64.68 \%$ of Sox $10^{+}$OLCs were met $^{+}$and this level of met $^{+}$ OLCs stayed roughly constant through $72 \mathrm{hpf}$, where $61.76 \%$ were met $^{+}$(Figure 2C). The consistent expression of met in the majority of OLCs from 55 to $72 \mathrm{hpf}$ is consistent with the major migratory period of OPCs, which occurs during this same time window. By
96 hpf, when most OLCs have completed their migration and many are initiating myelination of spinal cord axons, the population of OLCs that were met $^{+}$was decreased significantly to $8.56 \%$ (Figure 2C). These findings are consistent with previous investigations demonstrating that OPCs down-regulate expression of Met in order to differentiate into oligodendrocytes (Ohya et al. 2007). From these expression studies, we conclude that zebrafish OLCs express Met.

Because we observed Met expression in migratory OPCs, we next sought to more closely investigate the effect of MK2461 on their migration. First, we conducted a dose-response curve using the same treatment paradigm as the drug screen described above by treating $24 \mathrm{hpf}$ olig2:egfp zebrafish larvae with a 1\% DMSO control or increasing doses of MK2461 in 1\% DMSO and quantified 


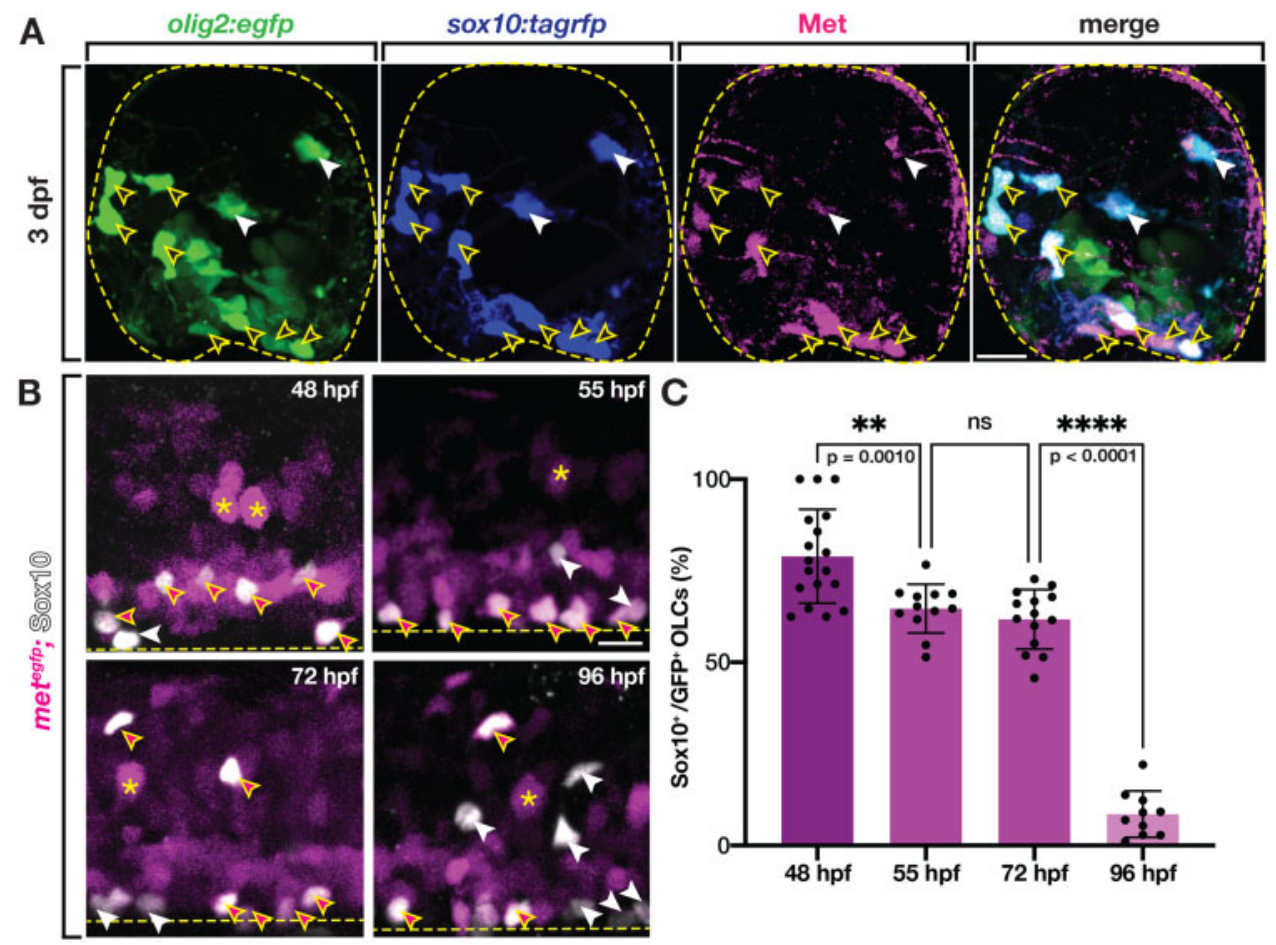

Figure 2 Zebrafish OLCs express Met. (A) Transverse section of 3 dpf sox10:tagrfp;olig2:egfp zebrafish spinal cord labeled with an antibody for Met (magenta). Yellow open arrowheads denote sox $10^{+} / \mathrm{Met}^{+}$OLCs. White arrowheads denote sox $10^{+} / \mathrm{Met}^{-}$OLCs. Yellow dashed circle denotes boundary of the spinal cord. (B) Lateral view of metegfp zebrafish spinal cords at 48, 55, 72, and 96 hpf labeled with a Sox10 antibody. Asterisks denote examples of $\mathrm{met}^{+}$motor neurons. Magenta-filled yellow arrowheads denote Sox $10^{+} / \mathrm{met}^{+}$OPCs, white arrowheads denote Sox $10^{+} / \mathrm{met}^{-}$ OLCs. Yellow dashed line denotes ventral edge of the spinal cord. (C) Percentage of OLCs that are Sox $10^{+}$and met:gfp in $322 \mu \mathrm{m}$ of spinal cord at 48 hpf $(n=18), 55 \mathrm{hpf}(n=12), 72 \mathrm{hpf}(n=14)$, and $96 \mathrm{hpf}(n=10)$. Mean with SEM. Statistical test: one-way ANOVA with Tukey's Multiple Comparison Test. Scale bars, $10 \mu \mathrm{m}(\mathrm{A}), 20 \mu \mathrm{m}$ (B).

the number of olig2 ${ }^{+}$OLCs in the dorsal spinal cord at $76 \mathrm{hpf}$. We found that increasing doses of MK2461 resulted in decreasing numbers of OLCs in the dorsal spinal cord compared to DMSOtreated larvae $(P<0.0001)$ (Figure $3 \mathrm{~A})$. To more directly assay the positioning and number of OLCs in control and drug-treated larvae, we used serial sectioning in 76 hpf olig2:egfp;sox10:mrfp zebrafish larvae treated with $1 \%$ DMSO or $10 \mu \mathrm{M}$ MK2461 in 1\% DMSO and quantified the number and location of olig $2^{+} /$sox $_{10} 0^{+}$ cells (Figure 3B). These studies revealed that the overall number of OLCs was reduced in the spinal cord of MK2461-treated larvae when compared to controls (Figure 3C), and the decrease affected both the number of dorsal and ventral OLCs $(P<0.0001)$ (Figure 3, $\mathrm{D}$ and $\mathrm{E})$. Interestingly, in these studies, we saw an increase in the number of OLCs in the pMN domain in larvae treated with MK2461, which indicates that OLCs were specified in MK2461treated larvae and that the migration defect we observed was not simply due to perturbed specification $(P=0.0349)$ (Figure $3 F$ ). Additionally, in contrast to our earlier studies in whole larvae where we observed that ventral OLCs were unaffected by MK2461 treatment, we saw a significant reduction in this population when we assessed their location in serial sections $(P<0.0001)$ (Figure 3E). We believe this occurred because it is difficult to observe individual ventral OLCs in whole-mount olig2:egfp larvae. Finally, the overall of reduction of OLCs in MK2461-treated larvae could be the result of reduced proliferation in OPCs, as Met is also implicated in regulating OPC proliferation in in vitro studies using OPC cell culture (Ohya et al. 2007), and we investigate this possibility later in this manuscript. Taken together, we conclude that Met mediates OPC migration and potentially OPC proliferation during development.

\section{Met is required for initial developmental OPC migration}

Given the migration phenotype we observed with the c-Met inhibitor, MK2461, and the expression pattern of Met in zebrafish OLCs, we sought to further investigate Met as a mediator of OPC migration during development using CRISPR/Cas9 mutagenesis that resulted in a 16 base pair insertion in the second exon of met that results in an early stop codon (Hruscha et al. 2013; Hwang et al. 2013) (Figure 4A). This mutation causes the premature termination in the beta chain of the sema domain resulting in a truncated polypeptide that would be functionally unable to homodimerize upon Hgf binding and therefore, would be unable to initiate downstream signaling (Figure 4B). Importantly, these mutant larvae had an overall normal morphology and body length when compared to wildtype siblings (Supplementary Figure S2A). To confirm the incorporation of a 16 base pair insertion, we isolated RNA from met $^{+/+}$, met $^{+/ u v a 38}$, and met ${ }^{\text {uva } 38 / u v a 38}$ larvae and performed RT-PCR. As expected, met ${ }^{u v a 38}$ heterozygous and homozygous larvae had a larger band compared to wildtype that was consistent with a 16 bp insertion (Supplementary Figure $\mathrm{S} 2 \mathrm{~B})$. Finally, to confirm our met allele contained a deleterious mutation in met, we crossed a heterozygous met ${ }^{\text {uva38 }}$ adult with a met $\mathrm{fh}^{\text {f534 }}$ heterozygous adult, which has a 25 base pair deletion which leads to a premature stop at amino acid 1105, and imaged the spinal cord of $72 \mathrm{hpf}$ olig2:egfp mutant larvae (Supplementary

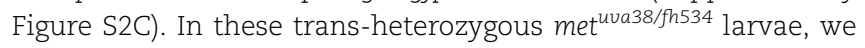
observed a significant decrease in the number of dorsal OLCs when compared with wildtype siblings (Supplementary Figure S2D). 
A

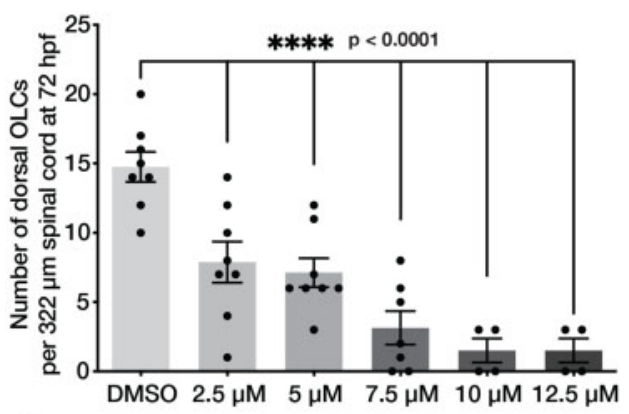

c

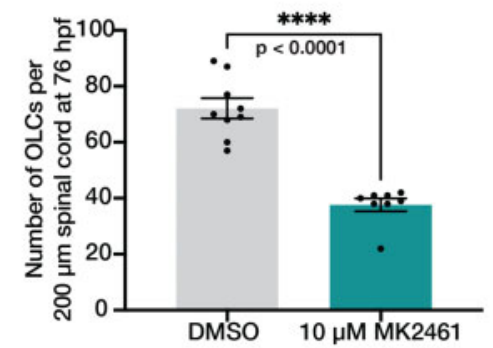

E

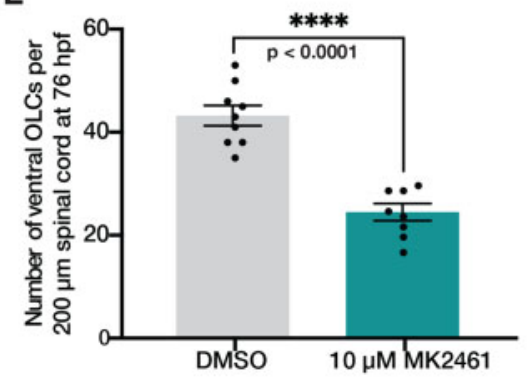

B

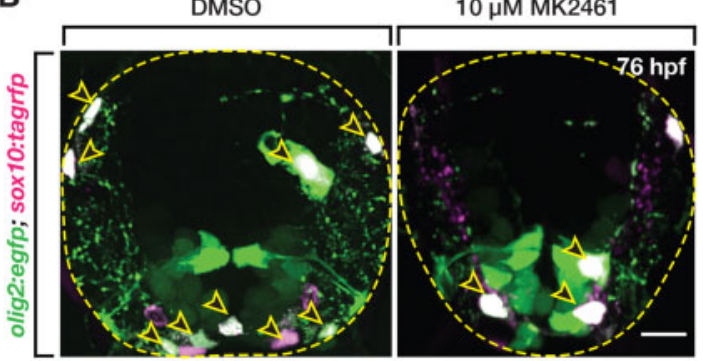

D

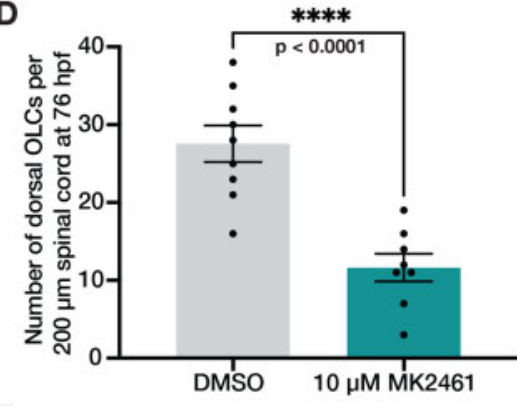

$\mathbf{F}$

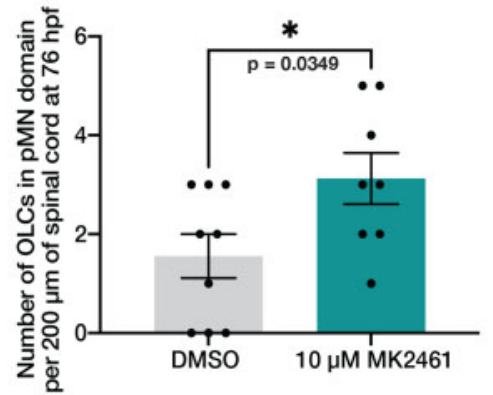

Figure 3 Met receptor inhibition decreases the number of OLCs in spinal cord. (A) Dose-response curve of the number of OLCs in the dorsal spinal cord of fish treated from $24 \mathrm{hpf}$ to $3 \mathrm{dpf}$ with $1 \%$ DMSO $(n=8)$ and $10 \mu \mathrm{M}$ MK2461 in $1 \%$ DMSO in the following doses: $2.5 \mu \mathrm{M}(n=8), 5 \mu \mathrm{M}(n=8), 7.5 \mu \mathrm{M}(n=7)$, $10 \mu \mathrm{M}(n=4)$, and $12.5 \mu \mathrm{M}(n=8)$. Statistical test: one-way ANOVA with Dunnett's Multiple Comparison Test. (B) Transverse sections of 76 hpf olig2:egfp;sox10:tagrfp larvae treated with DMSO or $10 \mu \mathrm{M}$ MK2461 from $24 \mathrm{hpf}$ to $3 \mathrm{dpf}$. Yellow open arrowheads denote sox10 $/ 0$ lig2 ${ }^{+}$OLCs. (C-F) Quantification of olig $2^{+} /$sox $10^{+}$OLCs from 10 sequential $20 \mu \mathrm{m}$ sections of olig2:egfp;sox10:tagrfp larvae treated with DMSO $(n=9)$ or MK2461 $(n=8)$ from $24 \mathrm{hpf}$ to $3 \mathrm{dpf}$. Mean with SEM. Student's t-test was used in C-F. Scale bar, $10 \mu \mathrm{m}$.

We next used this new met ${ }^{\text {uva3s }}$ allele to assess the role of met in regulating developmental OPC migration. Using in vivo, timelapse imaging in combination with our cell-tracking software in 55 hpf olig2:egfp; met $^{+/+}$, met ${ }^{+/ \text {uva } 38}$, and met $^{\text {uva }}$ (h8/uva38 larvae, we analyzed OPC migration (Wang et al. 2018). This imaging of oli-

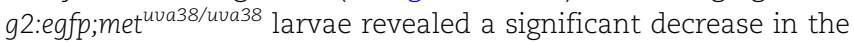
number of migrating OPCs compared to met $^{+/+}$larvae $(P=0.0307)$ (Figure 4, $C$ and D). Interestingly, the decrease in OPC migration in the met ${ }^{\text {uva }}$ 38/uva38 larvae primarily affected dorsally-migrating OLCs $(P=0.0207)$ (Figure $4 \mathrm{E})$. There was also a decrease in dorsally migrating OPCs in etet $^{+/ 4 v a 38}$ larvae, which is likely due to the tightly regulated nature of Met signaling $(P=0.0079)$ (Figure 4E) (Zhang and Babic 2015). In contrast, ventrally migrating OPCs in met $^{\text {uva38/uva38 }}$ larvae were similar to $\mathrm{met}^{+/+}$and $\mathrm{met}^{+/ \text {uva38 }}$ larvae, although there was a trend of reduced numbers of ventral migratory OPCs (Figure 4F). Finally, consistent with our Met inhibitor studies, OPCs showed no difference in average velocity of migration or distance traveled when met was perturbed (Supplementary Figure S3). Taken together, our results demonstrate that met is required for dorsal OPC migration during developmental OPC tiling.
To more closely examine the effect of a met mutation on developmental OPC migration, we performed serial sectioning on 76 hpf olig2:egfp;sox10:mrfp;met ${ }^{+/+}$, met $^{+/ \text {uva38}}$, and met tua $38 /$ uva38 $_{\text {lar- }}$ vae. We then imaged transverse sections of the spinal cord and assessed the location and number of OLCs (Figure 5A). Similar to what we observed with the c-Met inhibitor, we observed a decrease in the overall number of OLCs in met ${ }^{\text {uva38/uva38 }}$ larvae compared to wildtype siblings $(P=0.0137)$ (Figure 5B). Additionally, we observed a reduction in the number of OLCs in both the dorsal and ventral portions of the spinal cord in met uva $38 / u v a 38$ larvae compared to their wildtype siblings (dorsal, $P=0.0335$; ventral, $P=0.0014$ ) (Figure 5, C and D). This finding is slightly different than what we observed when looking at whole-mount, lateral views of the spinal cord in our met mutant larvae and larvae treated with MK2461. However, this difference is likely due to the fact that in lateral views, it is very difficult to visualize all ventral OLCs because of the expression of olig2:egfp in ventral spinal cord precursors and motor neurons. Therefore, our findings here with a careful analysis of OLC location in transverse sections is more accurate. Additionally, we observed an increase in the number of OLCs in the pMN domain in metuva38/uva38 larvae compared to 


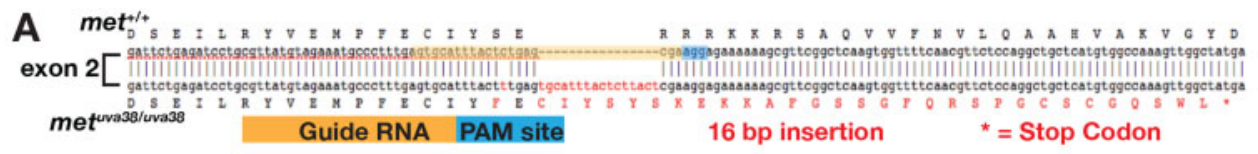

B met wildtype polypeptide: 1382 aa

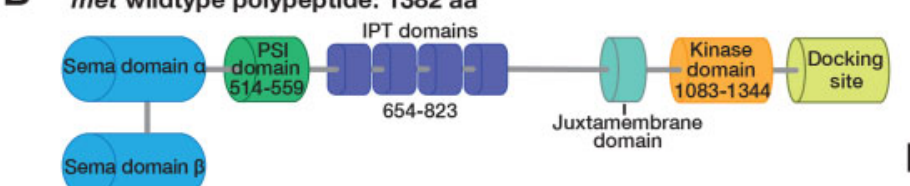

met ${ }^{\text {uvas3 }}$ mutant polypeptide: 328 aa

C

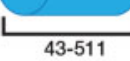

met $^{+/+}$
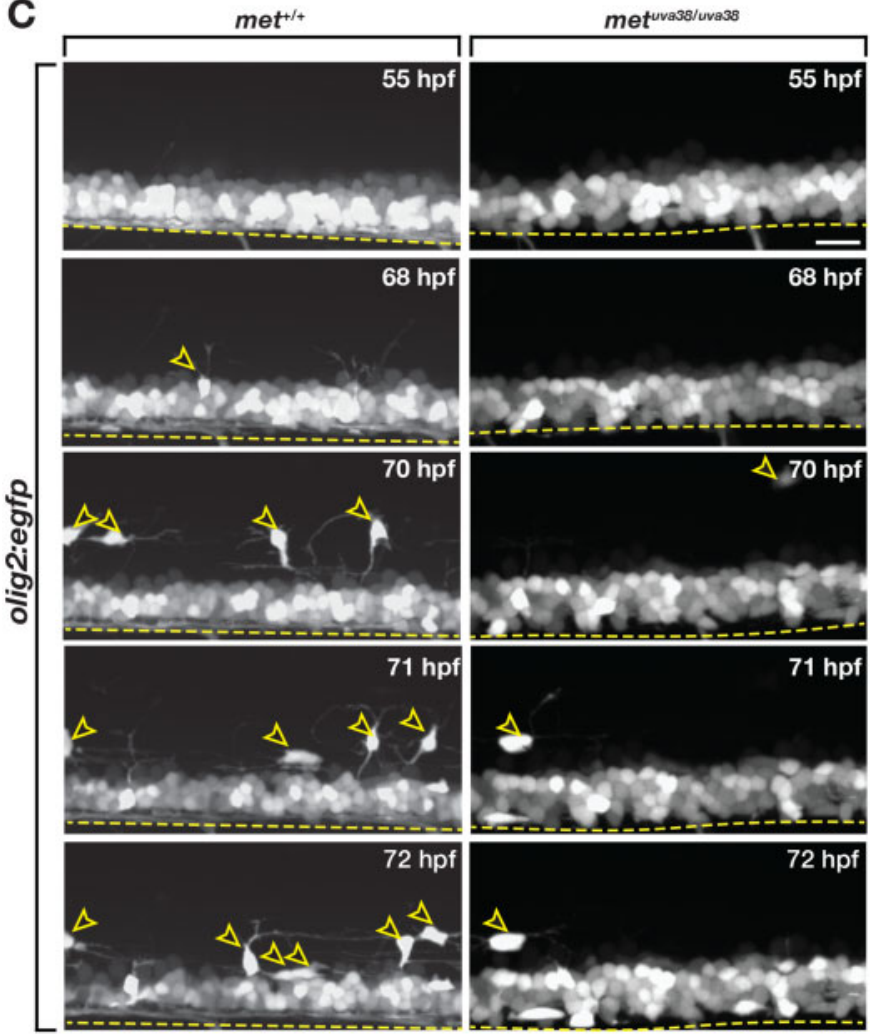

D
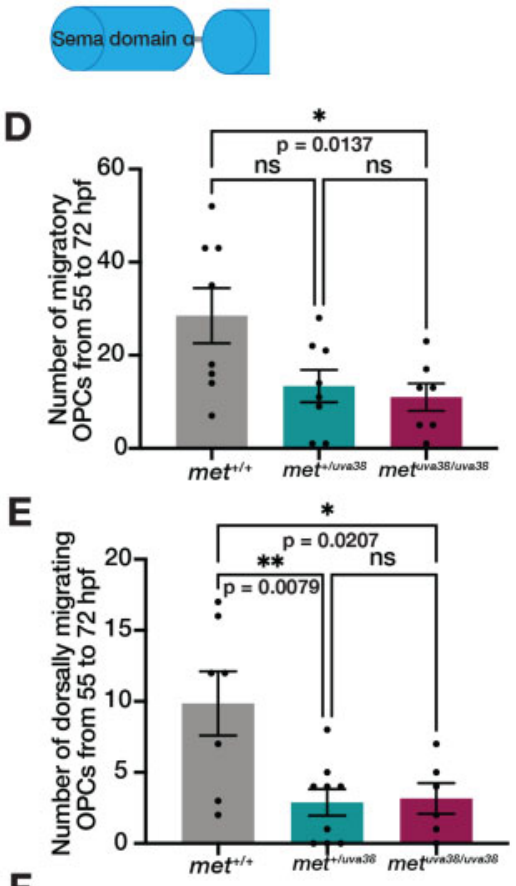

$\mathbf{F}$

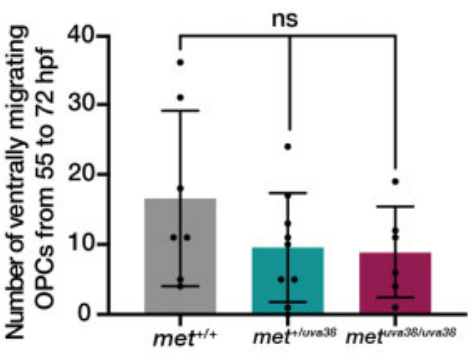

Figure 4 Met is required for initiation of dorsal OPC migration. (A) Diagram of met ${ }^{\text {tua38 }}$ mutant created using CRISPR/Cas9 mutagenesis with gRNA target sequence (yellow) and PAM site (blue) resulting in a 16 base pair insertion, which causes a frameshift mutation and early stop codon (asterisk). (B) Diagram of wildtype Met protein and Met mutant polypeptide sequences. (C) Images taken from $18 \mathrm{~h}$ time-lapse imaging of 55 hpf olig2:egfp met ${ }^{+/+}$, and met $^{\text {uva38/uva38 }}$ zebrafish larvae. Yellow open arrowheads denote dorsally migrating OPCs. Yellow dashed line denotes ventral edge of spinal cord. (D-F) Quantifications taken from time-lapse movies of 55 hpf olig2:egfp met ${ }^{+/+}(n=8)$, met ${ }^{+/ \text {uva38 }}(n=8)$, and met $\mathrm{t}^{\text {uva38/uva38 }}(n=6)$ zebrafish larvae. Mean with SEM. Statistical test: one-way ANOVA with Tukey's Multiple Comparison Test was used for D-F. Scale bar, $20 \mu \mathrm{m}$.

wildtype siblings, which is consistent with what we observed in larvae treated with MK2461 $(P=0.0013)$ (Figure 5E). This data also fits with what we observed in time-lapse movies of met mutant larvae, where we observed OPCs in the pMN domain of the spinal cord extend cellular processes into the dorsal spinal cord but then fail to migrate dorsally (Supplementary Movies S4 and S5). The increased number of OPCs in the pMN domain in met ${ }^{\text {uva38/ }}$ uva38 larvae compared to wildtype siblings, and the OPC process extension behavior in our time-lapse imaging indicate that there is a reduction in the number of OPCs that are migrating out of the pMN domain. This data supports our hypothesis that OPCs require met for initial migration out of the pMN domain during development, while sparing OPC specification.

Because Hgf is the ligand for the Met receptor, we wanted to investigate whether there would also be a reduction in dorsal OLC numbers in hgfa mutants as well (Isabella et al. 2020). To do this, we used Sox10 antibody labeling on 72 hpf hgfa mutant larvae and imaged lateral spinal cords and used optical sectioning to quantify the number of Sox $10^{+}$OLCs (Supplementary Figure $\mathrm{S} 4 \mathrm{~A})$. We hypothesized that we would see a similar change in OPC migration to what we observed in met mutant larvae. Excitingly, we observed a decrease in the overall number of OLCs in hgfa ${ }^{\text {fh528/fh528 larvae compared to wildtype siblings }(P=0.0008)}$ (Supplementary Figure S4B). Furthermore, we found that there was a significant decrease in the number of dorsal OPCs in hgfa ${ }^{f h 528 / f h 528}$ larvae compared to wildtype siblings $(P<0.0001)$, though the number of ventral OLCs was not significantly different compared to wildtype siblings (Supplementary Figure S4, C and D). Finally, we saw an increase in the number of OLCs in the pMN domain in hgfafh528/fh528 larvae compared to wildtype siblings $(P=0.0004)$ (Supplementary Figure S4E). These data are all consistent with what we observed in our met mutant larvae and 
A

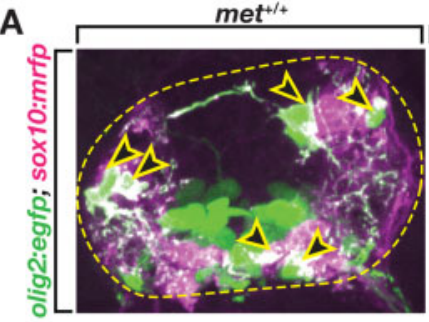

C

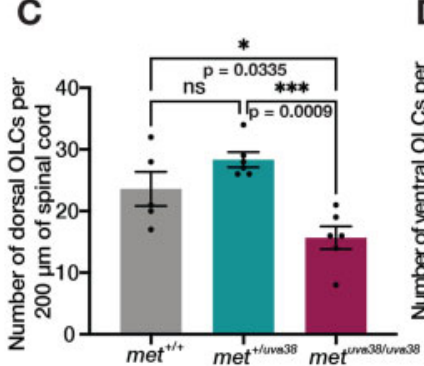

F $0 \mathrm{hpf}$

$70 \mathrm{hpf}$ met $^{\text {uva } 38 / u v a 38}$

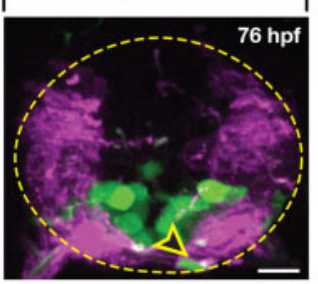

D
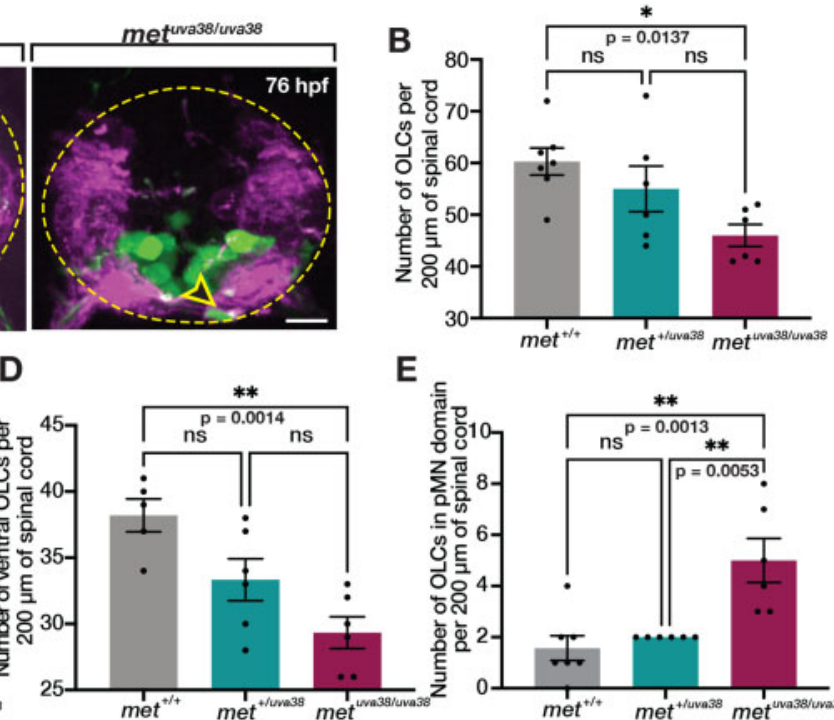

E
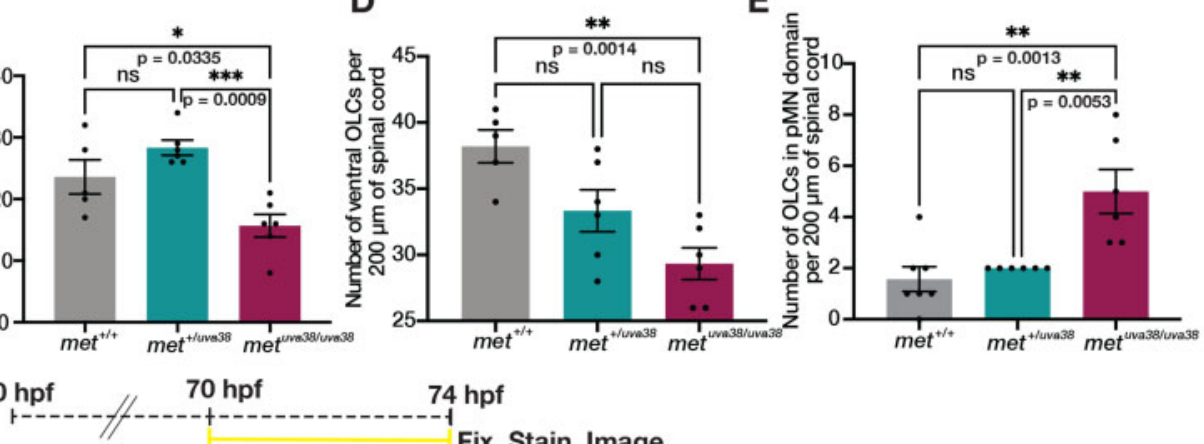

Fix, Stain, Image
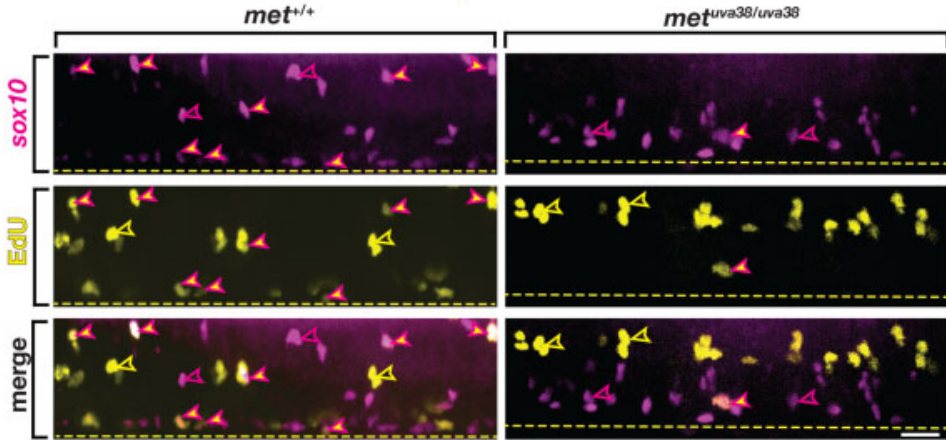

G

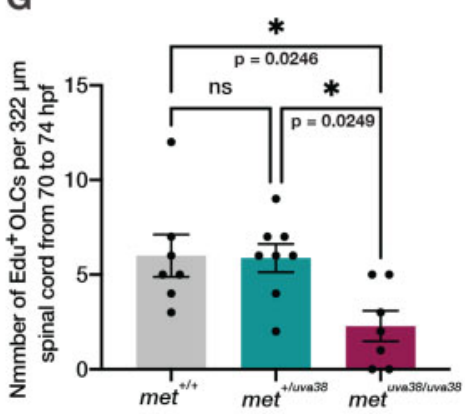

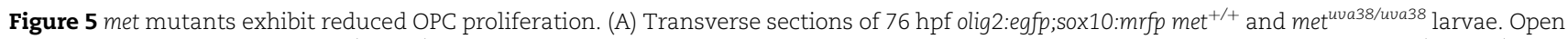
yellow arrowheads denote sox10 $/ 0$ lig2 $2^{+}$OPCs. Dashed yellow circle denotes boundary of the spinal cord. (B-E) Quantifications of olig2 $/$sox10 $0^{+}$OLCs from ten sequential $20 \mu \mathrm{m}$ sections of $76 \mathrm{hpf}$ olig2:egfp;sox10:mrfp met ${ }^{+/+}(n=7)$, met ${ }^{+/ \text {uva38 }}(n=6)$, and met ${ }^{\text {uva38/uva38 }}(n=6)$ larvae. Mean with SEM. Statistical test: one-way ANOVA with Tukey's Multiple Comparison Test. (F) EdU treatment paradigm and lateral view spinal cord images of 74 hpf sox10:mrfp; et $^{+/+}$and met ${ }^{\text {uva38/uva38 }}$ zebrafish larvae. Magenta-outlined yellow arrowheads denote sox10 ${ }^{+} /$EdU ${ }^{+}$OPCs. Magenta open arrowheads denote sox $10^{+} / \mathrm{EdU}^{-}$OLCs. Yellow open arrowheads denote sox10 $/ 0^{-}$EdU ${ }^{+}$cells. Yellow dashed line denotes ventral edge of the spinal cord. (G) Quantifications of sox10 $/ \mathrm{EdU}^{+}$OLCs from spinal cord images of $76 \mathrm{hpf}$ sox10:mrfp EdU labeled met $^{+/+}(n=7)$, met ${ }^{+/ u v a 38}(n=8)$, and met ${ }^{\text {uva38/uva38 }}(n=7)$ larvae. Mean with SEM. Statistical test: one-way ANOVA with Tukey's Multiple Comparison Test. Scale bars (A) $10 \mu \mathrm{m}$, (F) $20 \mu \mathrm{m}$.

further support that Met signaling is required for developmental OPC migration.

From these data, we hypothesize that the reduction in the

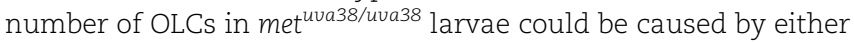
a reduction in OPC specification, which would lead to fewer OLCs in the spinal cord, or a reduction in OPC proliferation, which would result in wildtype numbers of OPCs during specification, but fewer OPCs during the migratory period. Met has been implicated in regulating cellular proliferation in a number of different cell types including hepatocytes, melanocytes, and other epithelial cell types (Tamagnone and Comoglio 1997; Prat et al. 1998; Viticchiè et al. 2015). To investigate if the overall decrease of OPCs

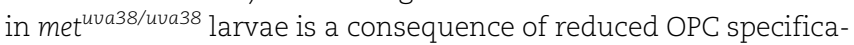
tion, we did serial sectioning of 48 hpf olig2:egfp;sox10:mrfp; met $^{+/+}$,

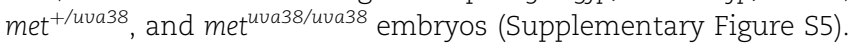
We chose $48 \mathrm{hpf}$ because it is sufficiently after the window of OPC specification which begins at $36 \mathrm{hpf}$, but prior to the main migratory period of OPCs which begins at approximately $55 \mathrm{hpf}$
(Kirby et al. 2006; Ravanelli et al. 2018). In these studies, we found that there was no difference in the number of OPCs in $\mathrm{met}^{+/+}$, met $^{+/ \text {uva38 }}$, and met ${ }^{\text {uva38/uva38 }}$ larvae at $48 \mathrm{hpf}$, which demonstrates that OPC specification is not affected by met mutation (Supplementary Figure S5).

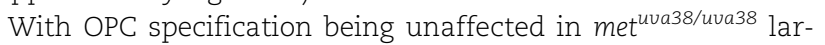
vae, we next hypothesized that the reduction in the number of

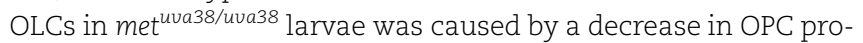
liferation, which has previously been demonstrated (Birchmeier and Gherardi 1998; Ohya et al. 2007; Viticchiè et al. 2015). To investigate OPC proliferation, we treated sox10:mrfp;met ${ }^{+/+}$, met $^{+/ \text {uva38, }}$ and metuva38/uva38 larvae with EdU (5-ethynyl-2'-deoxyuridine) from 70 to $74 \mathrm{hpf}$ in order to detect DNA synthesis in proliferative cells (Figure 5F). We then imaged spinal cords of EdU-treated larvae and quantified sox $10^{+} / \mathrm{EdU}^{+}$OLCs (Figure 5F). We observed that met ${ }^{\text {uva38/uva38 }}$ larvae had fewer EdU ${ }^{+}$OLCs compared to wildtype and heterozygous siblings at $74 \mathrm{hpf}$ (wt, $\mathrm{P}=0.0246$; heterozygous, $P=0.0249$ ) (Figure $5 G$ ). These results demonstrate that the 


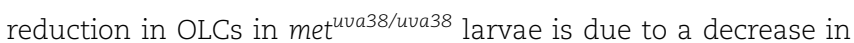
proliferation of OLCs and that met plays a role in regulating OLC proliferation and migration during development.

\section{Met inhibition in pre-migratory OPCs reduces dorsal migration out of the ventral spinal cord}

During development, Met is expressed in a number of developing central nervous system (CNS) neural populations, including motor neurons (Latimer and Jessen 2008). Because normal neuronal development influences OPC development (Ravanelli et al. 2018), we wanted to ensure that the phenotypes we observed in our inhibitor-treated and whole animal met mutant studies were not due to non-cell autonomous effects on OPCs. Therefore, we created cell lineage specific, Met dominant negative constructs to specifically perturb met signaling in OLCs.

To do this, we used site-directed mutagenesis to selectively mutate three critical tyrosines in the docking domain of the cMet receptor (Figure 6A), which, following Met dimerization, are trans-phosphorylated allowing for adaptor protein binding and downstream signaling (Figure 6B) (Soriano et al. 1995; Birchmeier and Gherardi 1998; Viticchiè et al. 2015). Previously published dominant negative Met (DNmet) constructs containing phenylalanine substitutions in the three docking site tyrosines of c-Met were successfully used in in vitro cell culture and zebrafish larvae for cell-specific reduction of Met signaling (Bardelli et al. 1999; Firon et al. 2000; Giordano et al. 2002; Latimer and Jessen 2008). The mutation of these tyrosines into phenylalanines physically prevents autophosphorylation of the docking site, thus preventing adaptor protein binding and downstream signaling (Ponzetto et al. 1996; Bardelli et al. 1999; Firon et al. 2000). We employed the same approach of creating point mutations that result in amino acid substitution of tyrosine to phenylalanine for the same critical tyrosines in the docking domain of the c-Met receptor as these previously published DNmet constructs (Firon et al. 2000; Giordano et al. 2002; Latimer and Jessen 2008). We confirmed successful mutation using Sanger sequencing, then drove our DNmet construct using either a sox10 or olig1 promoter and created stable transgenic lines (Figure 6D). In sox10:DNmet embryos and larvae, Met signaling is reduced in glial cells and OPCs upon their specification beginning at approximately $36 \mathrm{hpf}$ (Figure 6C). In olig1:DNmet embryos and larvae, Met signaling is reduced in OLCs at approximately $60 \mathrm{hpf}$, during their migratory phase (Auer et al. 2018) (Figure 6C). We additionally included an IRES:egfp reporter to genotype adults carrying either the sox10:DNmet ${ }^{+}$or olig1:DNmet ${ }^{+}$transgene (Figure 6D). Taken together, we used these DNmet transgenic animals to temporally control Met signaling in OLCs by modulating it in either premigratory OPCs using sox10:DNmet, or migratory OLCs using olig1:DNmet.

To investigate the effect of OPC-specific reduction of Met signaling, we used Sox10 antibody labeling to assess the position of OLCs in the spinal cord in sox10:DNmet and olig1:DNmet embryos and larvae by imaging lateral spinal cords and using optical sectioning to quantify cells (Figure 6E). At $55 \mathrm{hpf}$, we observed that the number of OPCs in both sox10:DNmet and olig1:DNmet larvae were unchanged compared to wildtype siblings (Figure 6F), supporting our conclusion that Met is not required for OPC specification. However, we observed decreased numbers of dorsal OPCs in sox10:DNmet larvae when compared to wildtype siblings at this stage $(P=0.0103)$ (Figure $6 G)$. In contrast, while the number of ventral OPCs was unchanged compared to wildtype and olig1:DNmet larvae (Figure 6H), the sox10:DNmet larvae exhibited a significant increase in the number of OPCs in the pMN domain compared to both wildtype $(P=0.0063)$ and olig1:DNmet larvae at 55 hpf $(P<0.0001)$ (Figure 6I). These results demonstrate a significant reduction in dorsal migration of OPCs out of the pMN domain when Met signaling is reduced in pre-migratory OPCs using the sox10:DNmet construct. Additionally, while the overall positioning of the OPCs in the spinal cord among the three groups at 55 hpf demonstrated a large population of ventral OPCs, sox10:DNmet larvae exhibited an expanded population of pMN domain OPCs that was significantly different from both wildtype $(P<0.0001)$ and olig1:DNmet larvae $(P<0.0001)$ (Figure 6, J and $K)$. These results demonstrate that inhibition of Met signaling in premigratory OPCs causes a significant shift in the distribution of the position of OPCs toward the pMN domain at $55 \mathrm{hpf}$, indicating that OPCs require Met signaling to migrate dorsally during development.

We wanted to further examine the effect of reduced Met signaling in OLCs by looking at OLC positioning in the spinal cord toward the end of the migratory period at $72 \mathrm{hpf}$. We used Sox10 antibody labeling to identify OLCs in the spinal cord and found that, at $72 \mathrm{hpf}$, the number of OLCs remained unchanged compared to wildtype in both the sox10:DNmet and olig1:DNmet zebrafish larvae (Figure 7, A and B). Interestingly, at $72 \mathrm{hpf}$, the number of dorsal OLCs was reduced in sox10:DNmet larvae compared to both wildtype $(P=0.0042)$ and olig1:DNmet $(P=0.0461)$ larvae (Figure 7C). Additionally, ventral OLCs were reduced in sox10:DNmet larvae compared to wildtype $(P=0.0286)$ (Figure 7D). Concordantly, the number of pMN domain OLCs in sox10:DNmet was significantly increased compared to both wildtype $(P<0.0001)$ and olig1:DNmet $(P<0.0001)$ larvae (Figure $7 E)$. Furthermore, the significantly increased percentage of OLCs in the pMN domain in sox10:DNmet larvae resulted in a reduced percentage of both dorsal and ventral OLCs in sox10:DNmet larvae compared to both wildtype and olig1:DNmet larvae at this stage (Figure 7, F and G). Additionally, olig1:DNmet larvae had a slightly increased percentage of pMN domain OLCs when compared to wildtype, though significantly less than sox10:DNmet $(P<0.0001)$, indicating that reducing Met signaling later in development can also affect OLC positioning $(P=0.0147)$ (Figure $7 G$ ). Taken together, these data demonstrate that reducing Met signaling specifically in pre-migratory OPCs causes a reduction in dorsal OPC migration following specification. Additionally, this reduction in migration is not observed when Met signaling is knocked down in OLCs that are already in the migratory window. Therefore, Met acts cell-autonomously to induce OPC migration, following specification, during developmental OPC tiling.

\section{Discussion}

OPCs are a group of highly motile and dynamic cells that are specified during early development. Following specification, they migrate rapidly to become evenly distributed throughout the CNS in a process termed tiling. While this process is described phenomenologically in the literature, there is still much left to be discovered about how tiling is molecularly regulated (Kirby et al. 2006; Hughes et al. 2013). Using an unbiased small molecule screen and various genetic approaches, we sought to identify molecular mediators of the initial phase of OPC tiling, dorsal migration. In our studies, we showed that Met, the receptor for Hgf, is required for dorsal OPC migration and OLC proliferation. Additionally, using cell-specific reduction of Met signaling, we show that Met is required for initial migration in pre-migratory OPCs, specifically. 

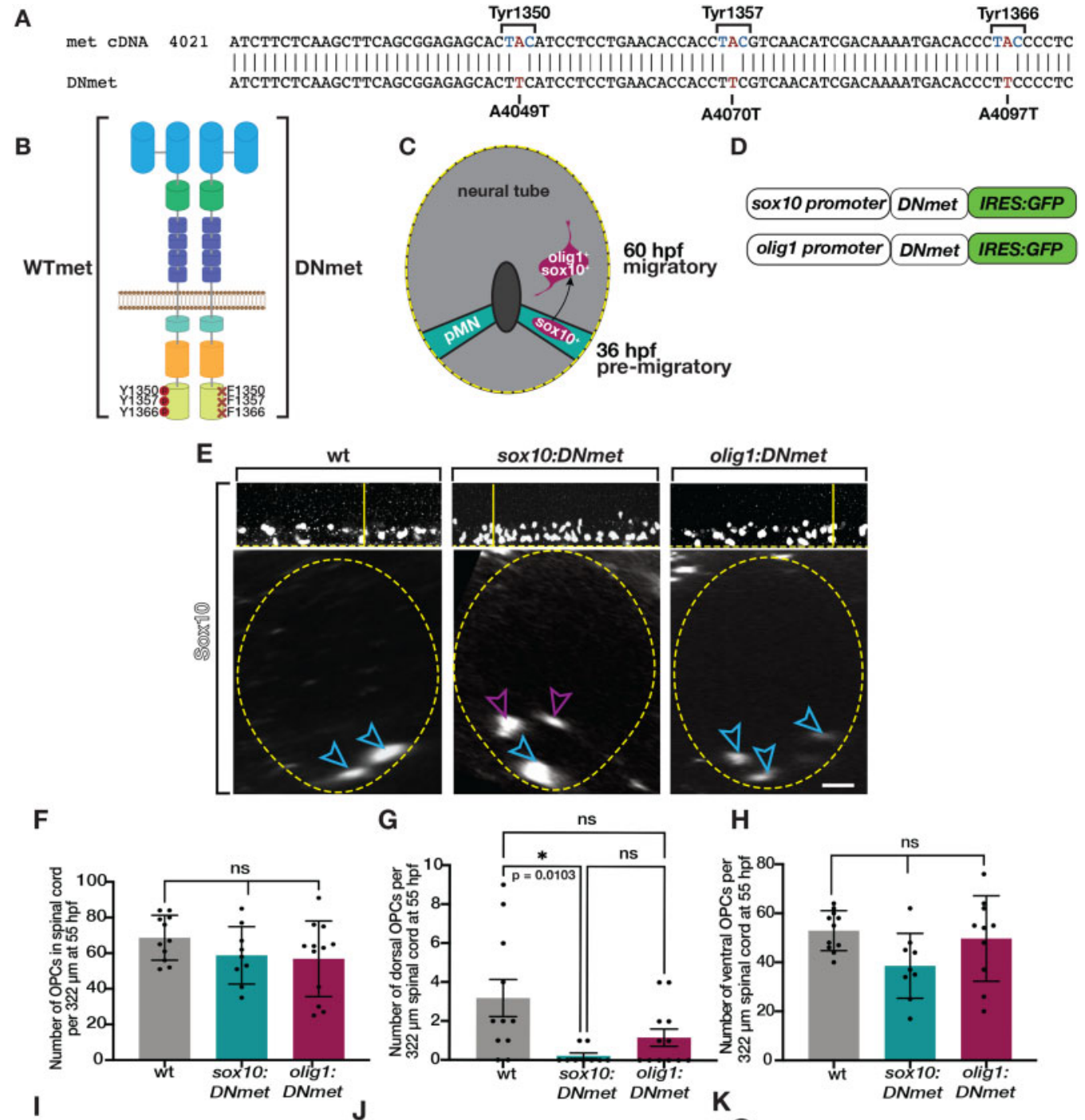

$\mathbf{F}$

G

ns

H
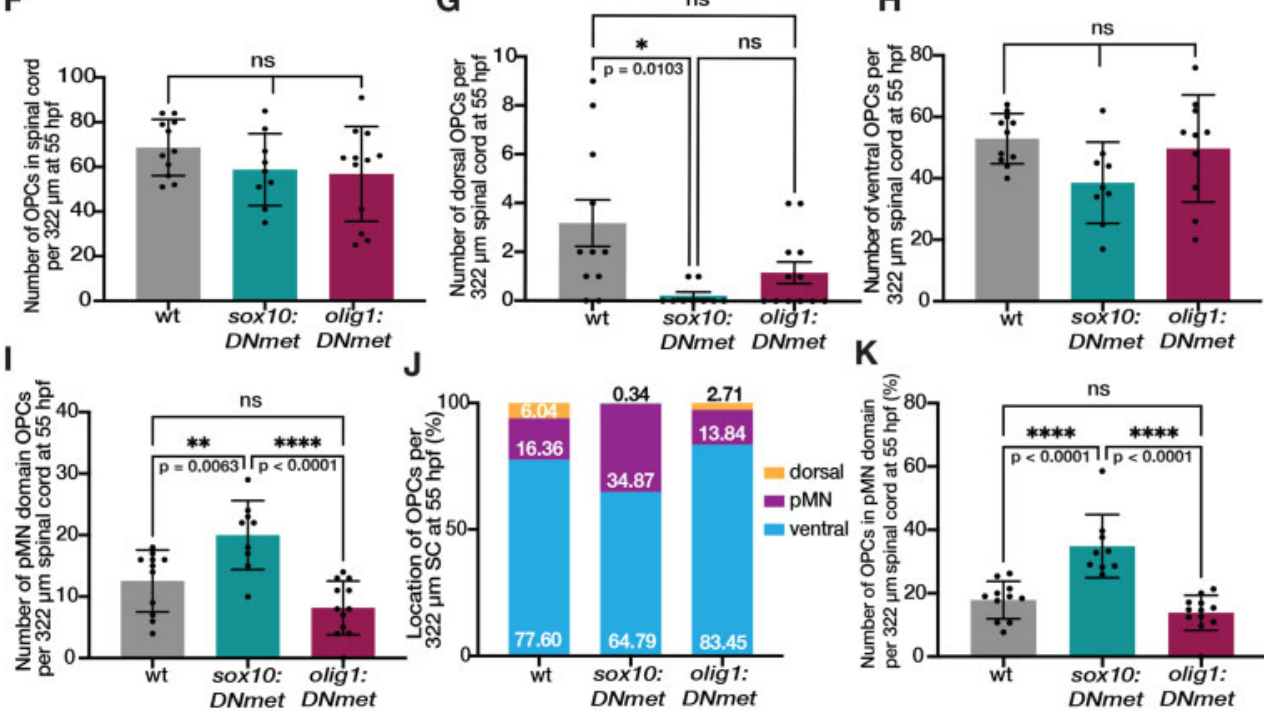

Figure 6 sox10:DNmet reduces OPC migration at 55 hpf. (A) Schematic of Met receptor showing site-specific mutations converting adenines (A) to thymines (T) resulting in amino acid changes of tyrosines (Y) to phenylalanines (F). (B) Diagram of amino acid substitutions in docking site tyrosines created using site-directed mutagenesis. (C) Diagram of developing neural tube showing sox10 turns on in pre-migratory OPCs around 36 hpf and olig 1 turns on in migratory OPCs around $60 \mathrm{hpf}$. (D) Schematic of DNmet constructs showing DNmet is driven by either a sox10 or olig1 promoter and includes IRES:GFP coding sequence. (E) Lateral (upper) and optical section (lower) views of Sox10 antibody labeled 55 hpf wildtype, sox10:DNmet, and olig1:DNmet larvae. Horizontal yellow line denotes location of orthogonal view shown below. Blue open arrowheads denote ventral OPCs. Purple open arrowheads denote pMN domain OPCs. Yellow dashed line denotes ventral edge of the spinal cord. Yellow dashed circle denotes spinal cord boundary. (F-K) Quantifications taken from images of $55 \mathrm{hpf}$ Sox10 antibody labeled wildtype $(n=10)$, sox10DN:met $(n=8)$, and olig1:DNmet ( $n=12)$ zebrafish spinal cords. Mean with SEM. Statistical test: one-way ANOVA with Tukeys's Multiple Comparison Test. Scale bar, $20 \mu \mathrm{m}$.

This work represents a significant advance in the understanding of Met signaling in OPC development because it is the first in vivo investigation demonstrating that loss of Met signaling reduces OPC migration. Previous in vitro studies demonstrated that applying Hgf to primary OPC cultures induced chemotaxis and proliferation (Kilpatrick et al. 2000; Lalive et al. 2005; Ohya et al. 2007; Mela and Goldman 2013). This in vitro data combined with our in vivo work demonstrates that Met expression on OPCs regulates their migration.

Interestingly, in our studies, we observed a trend in $\mathrm{met}^{+/ u v a 38}$ larvae where they occasionally exhibited OLC migration phenotypes similar to met mutants. A handful of studies have 

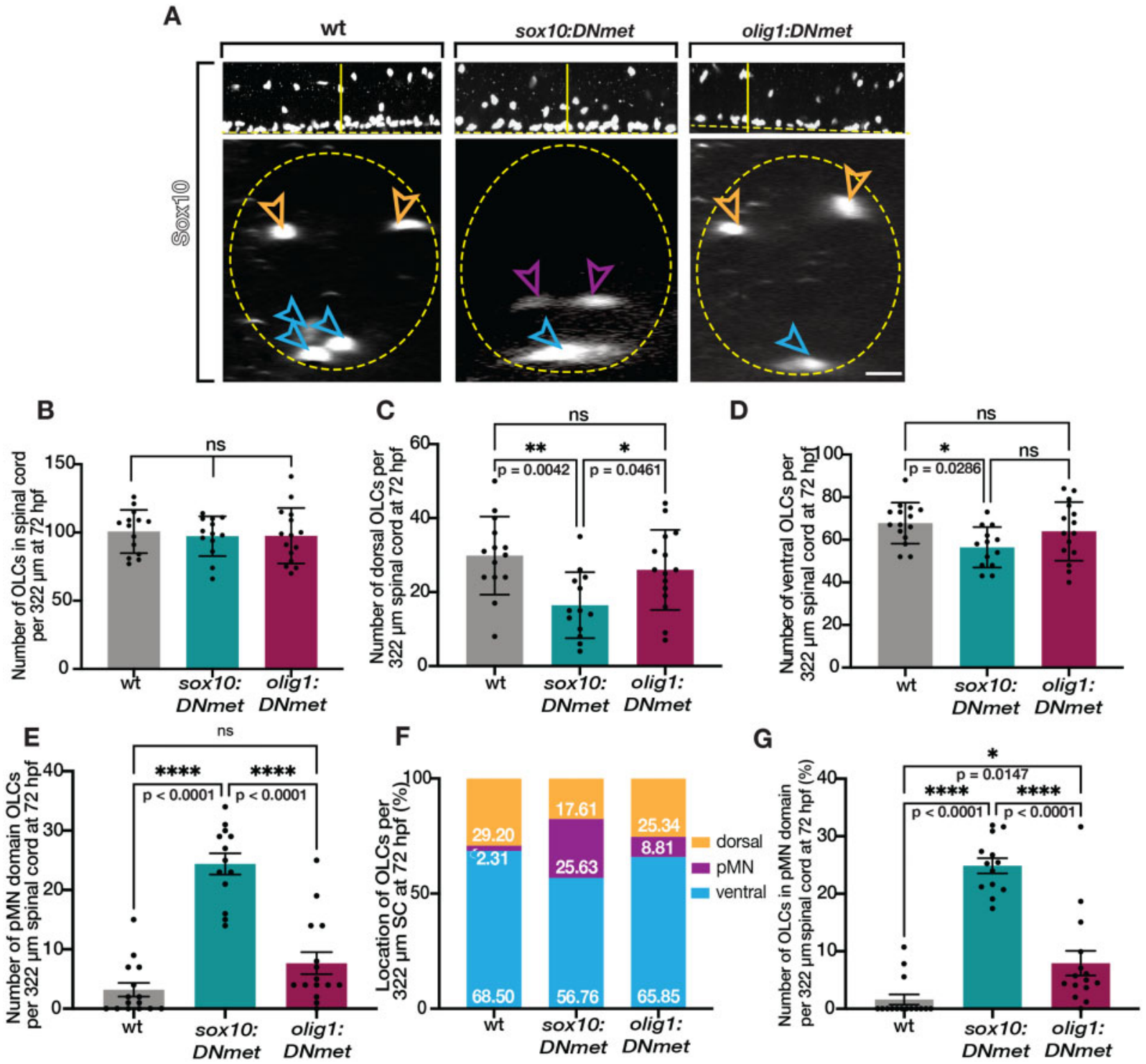

Figure 7 Met knock-down migration defects in pre-migratory OPCs persist to $72 \mathrm{hpf}$. (A) Lateral (upper) and optical section (lower) views of Sox10 antibody labeled $72 \mathrm{hpf}$ wildtype, sox10:DNmet, and olig1:DNmet zebrafish larvae. Horizontal yellow line denotes location of orthogonal view shown below. Orange open arrowheads denote dorsal OLCs. Purple open arrowheads denote pMN domain OLCs. Blue open arrowheads denote ventral OLCs. Yellow dashed line denotes ventral edge of the spinal cord. Yellow dashed circle denotes spinal cord boundary. (B-G) Quantifications taken from images of 72 hpf Sox10 antibody labeled wildtype $(n=14)$, sox10DN:met $(n=13)$, and olig1:DNmet $(n=15)$ zebrafish spinal cords. Mean with SEM. Statistical test: one-way ANOVA with Tukey's Multiple Comparison Test. Scale bar, $20 \mu \mathrm{m}$.

demonstrated that heterozygous met mutations can result in pathologically relevant outcomes (Ma et al. 2005; Matzke et al. 2007). More investigation into the regulation of Met signaling in OPCs and the normal physiological levels of active Met on OPCs would provide insight into the consequences of perturbing Met signaling in OPCs. Based on our observations here, it is likely that a certain threshold of Met signaling must be achieved for wildtype developmental processes to proceed and our heterozygous met mutants do not achieve this level of Met signaling resulting in the observed intermediate phenotypes.

What still remains unclear regarding the Met signaling pathway and OPC migration, is the source of Hgf. Some studies suggest that chemotropic factors released by astrocytes are influential in regulating OPC migration and positioning in the spinal cord (Tsai et al. 2002; Frost et al. 2009; Singh et al. 2019). Hgf/ Met signaling could be functioning in a similar way. RNA-seq datasets show that astrocytes express high levels of Hgf during development (Zhang et al. 2014; Lake et al. 2016). Further investigations into Hgf expression and signaling in combination with newly published tools to investigate astrocytes in zebrafish could provide a wider lens into how Hgf/Met signaling regulates OPC migration (Chen et al. 2020).

Beyond developmental tiling, OPCs remain evenly tiled throughout life and utilize similar processes of migration, proliferation, contact-mediated repulsion, and apoptosis to maintain their spacing (Hughes et al. 2013; Tsata et al. 2019). While a number of chemotactic molecules have been investigated in developmental OPC tiling, adult tiling and how it is maintained remains largely unknown. Another benefit of using zebrafish is that homozygous met mutants can live into adulthood and therefore, can be utilized to study these tiling processes in mature animals (Nord et al. 2019). It is possible that, while adult OPCs remain in 
their distinct domain with little migration, Met could be utilized in response to injury. For example, following the loss of OLCs, surviving OPCs proliferate and migrate into OLC-depleted regions (Kirby et al. 2006; Kang et al. 2010; Hughes et al. 2013; Birey and Aguirre 2015). Additionally, previous studies using the disease model for multiple sclerosis in mice, experimental autoimmune encephalomyelitis, demonstrate that macrophages and microglia release Hgf and OPCs upregulate Met signaling in response to demyelinated legions (Lalive et al. 2005; Moransard et al. 2009). Beyond these studies, little is known about how OPCs respond to injury and initiate migration and it is possible that OPCs utilize the same migration mechanisms in both development and injury-response. With so much still unknown about how OPC behaviors are molecularly-mediated, this work demonstrates the critical role that Met plays in regulating initial OPC migration during development. Future work will need to be done to identify how Met signaling is regulated in OPCs and what role, if any, Met plays in other OPC process, such as adult tiling and response to injury.

\section{Data availability}

Fish lines and plasmids are available upon request. The authors affirm that all data necessary for confirming the conclusions of the article are present within the article, figures, and table. Supplemental Material available at figshare: https://doi.org/10. $25387 / g 3.14988414$.

\section{Acknowledgements}

We thank Lori Tocke for zebrafish care and members of the Kucenas Lab for valuable discussions.

\section{Funding}

This work was funded by the NIH/National Institutes of Neurological Disorders and Stroke (NINDS): R01NS107525 (S.K.), F31NS108660 (M.F.A.), and R01NS109425 (C.B.M.); by the NIH/ National Institute of General Medical Sciences: T32GM008715 (M.F.A.); by the NIH/Eunice Kennedy Shriver National Institute of Child Health and Human Development: F32HD096860 (A.J.I.); and by the NIH/National Institute of Mental Health: R01MH11504 (G.Y.).

\section{Conflicts of interest}

The authors declare that there is no conflict of interest.

\section{Literature cited}

Ashton RS, Conway A, Pangarkar C, Bergen J, Lim K, et al. 2012. Astrocytes regulate adult hippocampal neurogenesis through ephrin-B signaling. Nat Neurosci. 15:1399-1406. doi:10. 1038/nn.3212.

Auer F, Vagionitis S, Czopka T. 2018. Evidence for myelin sheath remodeling in the CNS revealed by in vivo imaging. Curr Biol. 28: 549-559.e3. doi:10.1016/j.cub.2018.01.017.

Bardelli A, Longati P, Williams TA, Benvenuti S, Comoglio PM. 1999. A peptide representing the carboxyl-terminal tail of the Met receptor inhibits kinase activity and invasive growth. J Biol Chem. 274:29274-29281. doi:10.1074/jbc.274.41.29274.
Binari LA, Lewis GM, Kucenas S. 2013. Perineurial glia require notch signaling during motor nerve development but not regeneration. J Neurosci. 33:4241-4252. doi:10.1523/JNEUROSCI.4893-12.2013.

Birchmeier C, Gherardi E. 1998. Developmental roles of HGF/SF and its receptor, the c-Met tyrosine kinase. Trends Cell Biol. 8: 404-410. doi:10.1016/S0962-8924(98)01359-2.

Birey F, Aguirre A. 2015. Age-dependent netrin-1 signaling regulates NG2+ glial cell spatial homeostasis in normal adult gray matter. J Neurosci. 35:6946-6951. doi:10.1523/JNEUROSCI.0356-15.2015.

Cameron S, Rao Y. 2010. Molecular mechanisms of tiling and self-avoidance in neural development. Mol Brain. 3:28.doi: 10.1186/1756-6606-3-28.

Carney TJ, Dutton KA, Greenhill E, Delfino-Machín M, Dufourcq P, et al. 2006. A direct role for Sox10 in specification of neural crest-derived sensory neurons. Development. 133:4619-4630. doi: 10.1242/dev.02668.

Chen J, Poskanzer KE, Freeman MR, Monk KR. 2020. Live-imaging of astrocyte morphogenesis and function in zebrafish neural circuits. Nat Neurosci. 23:1297-1306. doi:10.1038/s41593-020-0703-X.

Chu T, Shields LBE, Zhang YP, Feng S-Q, Shields CB, et al. 2017 CXCL12/CXCR4/CXCR7 chemokine axis in the central nervous system: therapeutic targets for remyelination in demyelinating diseases. Neuroscientist. 23:627-648. doi:10.1177/1073858416685690.

Cunliffe VT, Casaccia-Bonnefil P. 2006. Histone deacetylase 1 is essential for oligodendrocyte specification in the zebrafish CNS. Mech Dev. 123:24-30. doi:10.1016/j.mod.2005.10.005.

De Biase LM, Schuebel KE, Fusfeld ZH, Jair K, Hawes IA, et al. 2017. Local cues establish and maintain region-specific phenotypes of basal ganglia microglia. Neuron. 95:341-356.e6. doi:10.1016/J.NEU RON.2017.06.020.

Dimou L, Simon C, Kirchhoff F, Takebayashi H, Götz M. 2008. Progeny of Olig2-expressing progenitors in the gray and white matter of the adult mouse cerebral cortex. J Neurosci. 28: 10434-10442.

Firon M, Shaharabany M, Altstock RT, Horev J, Abramovici A, et al. 2000. Dominant negative Met reduces tumorigenicity-metastasis and increases tubule formation in mammary cells. Oncogene. 19: 2386-2397. doi:10.1038/sj.onc.1203557.

Frost EE, Zhou Z, Krasnesky K, Armstrong RC. 2009. Initiation of oligodendrocyte progenitor cell migration by a PDGF-A activated Extracellular Regulated Kinase (ERK) signaling pathway. Neurochem Res. 34:169-181. doi:10.1007/s11064-008-9748-z.

Giordano S, Corso S, Conrotto P, Artigiani S, Gilestro G, et al. 2002. The semaphorin 4D receptor controls invasive growth by coupling with Met. Nat Cell Biol. 4:720-724. doi:10.1038/ncb843.

Grueber WB, Sagasti A. 2010. Self-avoidance and tiling: mechanisms of dendrite and axon spacing. Cold Spring Harb Perspect Biol. a001750.2:doi:10.1101/cshperspect.a001750.

Hruscha A, Krawitz P, Rechenberg A, Heinrich V, Hecht J, et al. 2013. Efficient CRISPR/Cas9 genome editing with low off-target effects in zebrafish. Development. 140:4982-4987. doi:10.1242/dev. 099085.

Huang W, Bhaduri A, Velmeshev D, Alvarez-Buylla A, Rowitch DH, Kriegstein AR, et al. 2020. Origins and proliferative states of human oligodendrocyte precursor cells. Cell. doi:10.1016/ j.cell.2020.06.027.

Hughes EG, Kang SH, Fukaya M, Bergles DE. 2013. Oligodendrocyte progenitors balance growth with self-repulsion to achieve homeostasis in the adult brain. Nat Neurosci. 16:668-676. doi: 10.1038/nn.3390.

Hwang WY, Fu Y, Reyon D, Maeder ML, Tsai SQ et al. 2013. Efficient genome editing in zebrafish using a CRISPR-Cas system. Nat Biotechnol. 31:227-229. doi:10.1038/nbt.2501. 
Isabella AJ, Barsh GR, Stonick JA, Dubrulle J, Moens CB. 2020. Retinoic acid organizes the zebrafish vagus motor topographic map via spatiotemporal coordination of Hgf/Met signaling. Dev Cell. 53:344-357.e5. doi:10.1016/j.devcel.2020.03.017.

Jarjour AA, Manitt C, Moore SW, Thompson KM, Yuh S-J, et al. 2003. Netrin-1 is a chemorepellent for oligodendrocyte precursor cells in the embryonic spinal cord. J Neurosci. 23:3735-3744.

Kang SH, Fukaya M, Yang JK, Rothstein JD, Bergles DE. 2010. NG2+ CNS glial progenitors remain committed to the oligodendrocyte lineage in postnatal life and following neurodegeneration. Neuron. 68:668-681. doi:10.1016/j.neuron.2010.09.009.

Kawakami K. 2004. Transgenesis and gene trap methods in zebrafish by using the Tol2 transposable element. Methods Cell Biol. 77: 201-222. doi:10.1016/s0091-679x(04)77011-9.

Kilpatrick TJ, Ortuño D, Bucci T, Lai C, Lemke G. 2000. Rat oligodendroglia express c-met and focal adhesion kinase, protein tyrosine kinases implicated in regulating epithelial cell motility. Neurosci Lett. 279:5-8. doi:10.1016/S0304-3940(99)00928-3.

Kimmel CB, Ballard WW, Kimmel SR, Ullmann B, Schilling TF. 1995. Stages of embryonic development of the zebrafish. Dev Dyn. 203: 253-310.

Kimura Y, Hisano Y, Kawahara A, Higashijima SI. 2014. Efficient generation of knock-in transgenic zebrafish carrying reporter/driver genes by CRISPR/Cas9-mediated genome engineering. Sci Rep. 6545.4:doi:10.1038/srep06545.

Kirby BB, Takada N, Latimer AJ, Shin J, Carney TJ, et al. 2006. In vivo time-lapse imaging shows dynamic oligodendrocyte progenitor behavior during zebrafish development. Nat Neurosci. 9: 1506-1511. doi:10.1038/nn1803.

Kucenas S, Takada N, Park HC, Woodruff E, Broadie K, et al. 2008. CNS-derived glia ensheath peripheral nerves and mediate motor root development. Nat Neurosci. 11:143-151. doi:10.1038/nn2025.

Kwan KM, Fujimoto E, Grabher C, Mangum BD, Hardy ME, et al. 2007. The Tol2kit: A multisite gateway-based construction kit forTol2 transposon transgenesis constructs. Dev Dyn. 236:3088-3099. doi:10.1002/dvdy.21343.

Labun K, Montague TG, Krause M, Torres Cleuren YN, Tjeldnes H, et al. 2019. CHOPCHOP v3: expanding the CRISPR web toolbox beyond genome editing. Nucleic Acids Res. 47:W171-W174. doi: 10.1093/nar/gkz365.

Lake BB, Ai R, Kaeser GE, Salathia NS, Yung YC, et al. 2016. Neuronal subtypes and diversity revealed by single-nucleus RNA sequencing of the human brain. Science. 352:1586-1590. doi:10.1126/science.aaf1204.

Lalive P, Paglinawan R, Biollaz G, Kappos E, Leone D, et al. 2005. TGF-beta-treated microglia induce oligodendrocyte precursor cell chemotaxis through the HGF-c-Met pathway. Eur J Immunol. 35:727-737. doi:10.1002/eji.200425430.

Latimer AJ, Jessen JR. 2008. hgf/c-met expression and functional analysis during zebrafish embryogenesis. Dev Dyn. 237: 3904-3915. doi:10.1002/dvdy.21794

López-Hidalgo M, Hoover WB, Schummers J. 2016. Spatial organization of astrocytes in ferret visual cortex. J Comp Neurol. 524: 3561-3576. doi:10.1002/cne.24015.

Lu QR, Yuk D, Alberta JA, Zhu Z, Pawlitzky I, et al. 2000. Sonic Hedgehog-regulated oligodendrocyte lineage genes encoding bHLH proteins in the mammalian central nervous system. Neuron. 25:317-329. doi:10.1016/S0896-6273(00)80897-1.

Ma PC, Jagadeeswaran R, Jagadeesh S, Tretiakova MS, Nallasura $\mathrm{V}$, et al. 2005. Functional expression and mutations of c-Met and its therapeutic inhibition with SU11274 and small interfering RNA in non-small cell lung cancer. Cancer Res. 65: 1479-1488.
Matzke A, Sargsyan V, Holtmann B, Aramuni G, Asan E, et al. 2007. Haploinsufficiency of c-Met in cd44-/- mice identifies a collaboration of CD44 and c-Met in vivo. Mol Cell Biol. 27:8797-8806. doi: 10.1128/mcb.01355-07.

Mela A, Goldman JE. 2013. CD82 blocks cMet activation and overcomes hepatocyte growth factor effects on oligodendrocyte precursor differentiation. J Neurosci. 33:7952-7960. doi: 10.1523/JNEUROSCI.5836-12.2013.

Moransard M, Sawitzky M, Fontana A, Suter T. 2009. Expression of the HGF receptor c-met by macrophages in experimental autoimmune encephalomyelitis. Glia. 58:559-571. doi:10.1002/ glia.20945.

Nichols EL, Green LA, Smith CJ. 2018. Ensheathing cells utilize dynamic tiling of neuronal somas in development and injury as early as neuronal differentiation. Neural Dev. 13:1-22. doi: 10.1186/s13064-018-0115-8.

Nord H, Dennhag N, Tydinger H, von Hofsten J. 2019. The zebrafish HGF receptor met controls migration of myogenic progenitor cells in appendicular development. Barsh GS, editor. PLoS One. 14:e0219259.doi:10.1371/journal.pone.0219259.

Ohya W, Funakoshi H, Kurosawa T, Nakamura T. 2007. Hepatocyte growth factor (HGF) promotes oligodendrocyte progenitor cell proliferation and inhibits its differentiation during postnatal development in the rat. Brain Res. 1147:51-65. doi: 10.1016/J.BRAINRES.2007.02.045.

Peterson SM, Freeman JL. 2009. RNA isolation from embryonic zebrafish and cDNA synthesis for gene expression analysis. J Vis Exp. 1470.doi:10.3791/1470.

Ponzetto C, Zhen Z, Audero E, Maina F, Bardelli A, et al. 1996. Specific uncoupling of GRB2 from the met receptor: differential effects on transformation and motility. J Biol Chem. 271:14119-14123. doi: 10.1074/jbc.271.24.14119.

Prat M, Crepaldi T, Pennacchietti S, Bussolino F, Comoglio PM. 1998. Agonistic monoclonal antibodies against the Met receptor dissect the biological responses to HGF. J Cell Sci. 111:237-247.

Ravanelli AM, Kearns CA, Powers RK, Wang Y, Hines JH, et al. 2018. Sequential specification of oligodendrocyte lineage cells by distinct levels of Hedgehog and Notch signaling. Dev Biol. 444: 93-106. doi:10.1016/j.ydbio.2018.10.004.

Shin J, Park H-C, Topczewska JM, Mawdsley DJ, Appel B. 2003. Neural cell fate analysis in zebrafish using olig2 BAC transgenics. Methods Cell Sci. 25:7-14. doi:10.1023/B:MICS.0000006847. 09037.3a.

Singh J, Sharma K, Frost EE, Pillai PP. 2019. Role of PDGF-A-activated ERK signaling mediated FAK-paxillin interaction in oligodendrocyte progenitor cell migration. J Mol Neurosci. 67:564-573. doi: 10.1007/s12031-019-1260-1.

Soriano JV, Pepper MS, Nakamura T, Orci L, Montesano R. 1995. Hepatocyte growth factor stimulates extensive development of branching duct-like structures by cloned mammary gland epithelial cells. J Cell Sci. 108:413-430.

Spassky N, de Castro F, Le Bras B, Heydon K, Oise Qué Raud-LeSaux $F$, et al. 2002. Directional guidance of oligodendroglial migration by class 3 semaphorins and netrin-1. J Neurosci. 22:5992-6004.

Talbot JC, Amacher SL. 2014. A streamlined CRISPR pipeline to reliably generate zebrafish frameshifting alleles. Zebrafish. 11: 583-585. doi:10.1089/zeb.2014.1047.

Talbot JC, Teets EM, Ratnayake D, Duy PQ, Currie PD, et al. 2019. Muscle precursor cell movements in zebrafish are dynamic and require six family genes. Dev. 146:dev171421. doi: 10.1242/dev.171421.

Tamagnone L, Comoglio PM. 1997. Control of invasive growth by hepatocyte growth factor (HGF) and related scatter factors. 
Cytokine Growth Factor Rev. 8:129-142. doi:10.1016/S1359-6 101(97)00007-5.

Truett GE, Heeger P, Mynatt RL, Truett AA, Walker JA, et al. 2000. Preparation of PCR-quality mouse genomic DNA with hot sodium hydroxide and tris (HotSHOT). Biotechniques. 29:52-54. doi: 10.2144/00291bm09.

Tsai H-H, Frost E, To V, Robinson S, Ffrench-Constant C, et al. 2002. The chemokine receptor CXCR2 controls positioning of oligodendrocyte precursors in developing spinal cord by arresting their migration. Cell. 110:373-383. doi:10.1016/S00928674(02)00838-3.

Tsata V, Kroehne V, Reinhardt S, El-Armouche A, Brand M, et al. 2019. Electrophysiological properties of adult zebrafish oligodendrocyte progenitor cells. Front Cell Neurosci. 13:102.doi: 10.3389/fncel.2019.00102.

Villar-Cerviño V, Molano-Mazón M, Catchpole T, Valdeolmillos M, Henkemeyer M, et al. 2013. Contact repulsion controls the dispersion and final distribution of Cajal-Retzius cells. Neuron. 77:457-471. doi:10.1016/j.neuron.2012.11.023.

Viticchiè G, Muller P, Viticchiè G, Muller PAJ. 2015. c-Met and other cell surface molecules: interaction, activation and functional consequences. Biomedicines. 3:46-70. doi:10.3390/biomedicines3010046.
Wang C, Yizhi W, Yinxue W, Wu C-T, Yu G. 2019. muSSP: efficient min-cost flow algorithm for multi-object tracking. Advances in Neural Information Processing Systems, 32:425-434.

Wang Y, Ali M, Yue W, Kucenas S, Yu G. 2018. Detection and tracking of migrating oligodendrocyte progenitor cells from in vivo fluorescence time-lapse imaging data. Proc IEEE Int Symp Biomed Imaging. 2018:961-964.

Wang Y, Wang C, Ranefall P, Broussard GJ, Wang Y, et al. 2020. SynQuant: An automatic tool to quantify synapses from microscopy images. Bioinformatics. 36:1599-1606. doi:10.1093/bioinformatics/btz760.

Zhang J, Babic A. 2015. Regulation of the MET oncogene: molecular mechanisms. Carcinogenesis. 37:345-355. doi:10.1093/carcin/ bgw015.

Zhang Y, Chen K, Sloan SA, Bennett ML, Scholze AR, et al. 2014. An RNA-sequencing transcriptome and splicing database of glia, neurons, and vascular cells of the cerebral cortex. J Neurosci. 34: 11929-11947. doi:10.1523/JNEUROSCI.1860-14.2014.

Zhu Y, Crowley SC, Latimer AJ, Lewis GM, Nash R, et al. 2019. Migratory neural crest cells phagocytose dead cells in the developing nervous system. Cell. 179:74-89.e10. doi:10.1016/j.cell.2019.08.001.

Communicating editor: A. McCallion 